\title{
AN ADAPTIVE FINITE ELEMENT DTN METHOD FOR THE ELASTIC WAVE SCATTERING BY BIPERIODIC STRUCTURES
}

\author{
Gang BaO ${ }^{1}$, Xue Jiang ${ }^{2}$, PeiJun Li ${ }^{3, *}$ And XiaOkaI Yuan ${ }^{1,4}$
}

\begin{abstract}
Consider the scattering of a time-harmonic elastic plane wave by a bi-periodic rigid surface. The displacement of elastic wave motion is modeled by the three-dimensional Navier equation in an unbounded domain above the surface. Based on the Dirichlet-to-Neumann (DtN) operator, which is given as an infinite series, an exact transparent boundary condition is introduced and the scattering problem is formulated equivalently into a boundary value problem in a bounded domain. An a posteriori error estimate based adaptive finite element DtN method is proposed to solve the discrete variational problem where the DtN operator is truncated into a finite number of terms. The a posteriori error estimate takes account of the finite element approximation error and the truncation error of the DtN operator which is shown to decay exponentially with respect to the truncation parameter. Numerical experiments are presented to illustrate the effectiveness of the proposed method.
\end{abstract}

Mathematics Subject Classification. 78A45, 65N30, 65N12, 65N50.

Received January 17, 2021. Accepted November 3, 2021.

\section{INTRODUCTION}

This paper concerns the scattering of a time-harmonic elastic plane wave by a bi-periodic surface in three dimensions. Due to the wide and significant applications in seismology and geophysics, the elastic wave scattering problems have received ever increasing attention in both mathematical and engineering communities $[1,2,33]$. Compared with the acoustic and electromagnetic wave scattering problems, the elastic wave scattering problems are less studied due to the fact that the elastic wave consists of coupled compressional and shear wave components with different wavenumbers, which makes the analysis of the problems more complicated. In addition, there are two challenges for the elastic surface scattering problem: the solution may have singularity due to a possible non-smooth surface; the problem is imposed in an unbounded domain. In this paper, we intend to address both of these two issues by proposing an a posteriori error estimate based adaptive finite element method with the transparent boundary condition.

The a posteriori error estimates are computable quantities from numerical solutions. They can be used to measure the solution errors of discrete problems without requiring any a priori information of exact solutions

Keywords and phrases. Elastic wave equation, scattering by biperiodic structures, adaptive finite element method, transparent boundary condition, DtN map, a posteriori error estimate.

1 School of Mathematical Sciences, Zhejiang University, Hangzhou 310027, P.R. China.

2 Faculty of Science, Beijing University of Technology, Beijing 100124, P.R. China.

3 Department of Mathematics, Purdue University, West Lafayette, IN 47907, USA.

4 School of Mathematics, Jilin University, Changchun, Jilin 130012, China.

* Corresponding author: lipeijun@math.purdue.edu 
$[4,34]$. Since the a posteriori error estimate based adaptive finite element method has the ability to control the error and to asymptotically optimize the approximation, it is crucial for mesh modification such as refinement and coarsening $[19,35,38]$. The method has become an important numerical tool for solving boundary value problems of partial differential equations, especially for those where the solutions have singularity or multiscale phenomena.

The key of overcoming the second issue is to reformulate the unbounded domain problem into a boundary value problem in a bounded domain without generating artificial wave reflection. One possible approach is to make use of the perfectly matched layer (PML) techniques. The basic idea of the PML is to surround the domain of interest by a layer of finite thickness of fictitious medium that may attenuate the waves propagating from inside of the computational domain. When the waves reach the outer boundary of the PML region, their amplitudes are so small that the homogeneous Dirichlet boundary condition can be imposed. Due to the effectiveness and simplicity, since Bérenger proposed the technique to solve the time domain Maxwell equations [7], it has undergone a tremendous development of designing various PML methods to solving a wide range of unbounded domain scattering problems $[5,8,9,16-18,22,23,30]$. Combined with adaptive finite element methods, the PML method has been investigated to solve the two- and three-dimensional obstacle scattering problems $[12,13,15]$ and the two- and three-dimensional diffraction grating problems $[6,14,26]$. The a posteriori error estimates based adaptive finite element PML methods take account of the finite element discretization errors and the PML truncation errors which decay exponentially with respect to the PML parameters.

Alternatively, another effective approach to truncate the unbounded domain is to construct the Dirichlet-toNeumann (DtN) map and introduce the transparent boundary condition to enclose the domain of interest [21]. Since the DtN operator is exact, the transparent boundary condition can be imposed on the boundary which is chosen as close as possible to the scattering structure. Compared to the PML method, the DtN method can reduce the size of the computational domain. As a viable alternative to the PML method, the adaptive finite element DtN methods have also been developed recently to solve many two- and three-dimensional scattering problems, such as the acoustic scattering problems [25,27,39], the three-dimensional electromagnetic scattering problem [29], and the two-dimensional elastic wave scattering problems [31,32].

It is worth mentioning that the scattering problems by unbounded surfaces can also be solved by using integral equation based methods such as the boundary variation method [10,11] and the integral equation method [40]. One of the advantages of these methods is that the radiation condition is satisfied automatically in the integral formulation. But it may be difficult to handle the problem with a nonsmooth boundary or an inhomogenous medium.

This paper concerns the numerical solution of the elastic wave scattering by biperiodic structures in three dimensions. It is a non-trivial extension of the elastic wave scattering by periodic structures in two dimensions [32]. There are two challenges for the three-dimensional problem. First, the Helmholtz decomposition of the elastic wave equation gives two two-dimensional Helmholtz equations in the two-dimensional case; however, for the Helmholtz decomposition in the three-dimensional case, we have to consider a three-dimensional Helmholtz equation and a three-dimensional Maxwell equation, which makes the analysis much more complicated. Second, from the computational point of view, it is much more time-consuming to solve the three-dimensional problem than to solve the two-dimensional problem.

Specifically, we consider the scattering of a time-harmonic plane elastic wave by a biperiodic rigid surface. The elastic wave propagation is modeled by the three-dimensional Navier equation in the unbounded domain above the scattering surface. By the Helmholtz decomposition, a DtN operator is constructed in terms of Fourier series expansions for the compressional and shear wave components, then an exact transparent boundary condition is introduced to reduce the unbounded domain problem into an equivalent boundary value problem in a bounded domain. The nonlocal DtN operator needs to be truncated into a sum of finitely many terms in actual computation. However, it is known that the convergence of the truncated DtN operator could be arbitrary slow to the original DtN operator in the operator norm [24]. By carefully examining the properties of the exact solution, we observe that the truncated DtN operator converges exponentially to the original DtN operator when acting on the solution of the elastic wave equation, which enables the analysis of exponential convergence for this 


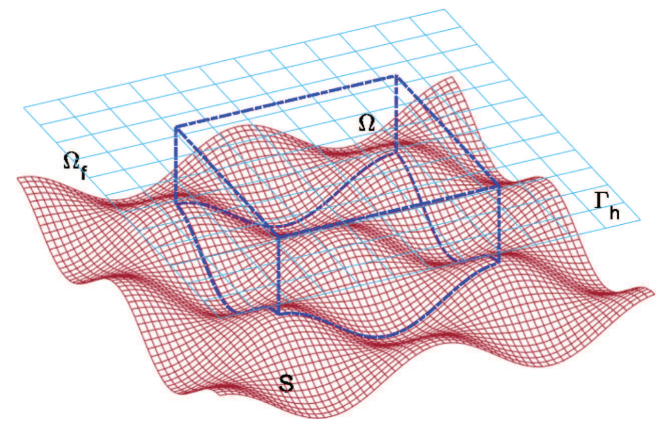

FiguRE 1. Problem geometry of the elastic scattering by a biperiodic surface.

work. Combined with the truncated DtN operator and finite element method, the discrete problem is studied. We develop a new duality argument to deduce the a posteriori error estimate. The a posteriori error estimate takes account of the finite element approximation error and the DtN operator truncation error which is shown to decay exponentially with respect to the truncation parameter $N$. Moreover, an a posteriori error estimate based adaptive finite element algorithm is presented to solve the discrete problem, where the estimate is used to design the algorithm to choose elements for refinements and to determine the truncation parameter $N$. Due to the exponential convergence of the truncated DtN operator, the choice of the truncation parameter $N$ turns out not to be sensitive to the given tolerance of accuracy. Numerical examples are presented to demonstrate the effectiveness of the proposed method.

The paper is organized as follows. In Section 2, the model equation is introduced for the scattering problem. Section 3 concerns the variational problem. By the Helmholtz decomposition, the DtN operator is constructed and the transparent boundary condition is introduced to reformulate the scattering problem into a boundary value problem in a bounded domain, and the corresponding weak formulation is presented. In Section 4, the discrete problem is studied by using the finite element method with the truncated DtN operator. Section 5 is devoted to the a posteriori error analysis for the discrete problem and the exponential convergence is proved for the truncated DtN operator. In Section 6, an adaptive finite element algorithm is described and numerical experiments are carried out to illustrate the competitive behavior of the proposed method. The paper is concluded with some general remarks and directions for future work in Section 7 .

\section{Problem Formulation}

Consider the scattering of a time-harmonic plane elastic wave by a rigid biperiodic surface. Due to the biperiodic structure, the scattering problem can be restricted into a single biperiodic cell, as shown in Figure 1. Let

$$
S=\left\{\boldsymbol{x}=\left(x_{1}, x_{2}, x_{3}\right)^{\top} \in \mathbb{R}^{3}:\left(x_{1}, x_{2}\right) \in\left(0, \Lambda_{1}\right) \times\left(0, \Lambda_{2}\right), x_{3}=f\left(x_{1}, x_{2}\right)\right\}
$$

be the scattering surface, where $f$ is a Lipschitz continuous biperiodic function with periods $\Lambda_{1}$ and $\Lambda_{2}$ in the $x_{1}$ and $x_{2}$ directions, respectively. Denote the open space above $S$ by

$$
\Omega_{f}=\left\{\boldsymbol{x} \in \mathbb{R}^{3}:\left(x_{1}, x_{2}\right) \in\left(0, \Lambda_{1}\right) \times\left(0, \Lambda_{2}\right), x_{3}>f\left(x_{1}, x_{2}\right)\right\},
$$

which is assumed to be filled with an isotropic and homogeneous elastic medium. The medium can be characterized by the Lamé parameters $\lambda, \mu$ and the mass density $\rho$ which is assumed to be unit for simplicity. Furthermore, we assume that the Lamé constants satisfy $\mu>0, \lambda+\mu>0$. Define

$$
\Gamma_{h}=\left\{\boldsymbol{x} \in \mathbb{R}^{3}:\left(x_{1}, x_{2}\right) \in\left(0, \Lambda_{1}\right) \times\left(0, \Lambda_{2}\right), x_{3}=h\right\},
$$


where $h$ is a constant satisfying $h>\max _{\left(x_{1}, x_{2}\right) \in\left(0, \Lambda_{1}\right) \times\left(0, \Lambda_{2}\right)} f\left(x_{1}, x_{2}\right)$. Denote by $\Omega$ the bounded domain enclosed by $S$ and $\Gamma_{h}$, i.e.,

$$
\Omega=\left\{\boldsymbol{x} \in \mathbb{R}^{3}:\left(x_{1}, x_{2}\right) \in\left(0, \Lambda_{1}\right) \times\left(0, \Lambda_{2}\right), f\left(x_{1}, x_{2}\right)<x_{3}<h\right\} .
$$

Let a compressional plane wave

$$
\boldsymbol{u}^{\mathrm{inc}}(\boldsymbol{x})=\boldsymbol{q} e^{\mathrm{i} \kappa_{1} \boldsymbol{q} \cdot \boldsymbol{x}}
$$

be sent from the above to impinge the surface, where

$$
\boldsymbol{q}=\left(\sin \theta_{1} \cos \theta_{2}, \sin \theta_{1} \sin \theta_{2},-\cos \theta_{1}\right)^{\top},
$$

$\theta_{1} \in[0, \pi / 2)$ and $\theta_{2} \in[0,2 \pi]$ are the incident angles, and $\kappa_{1}=\omega / \sqrt{\lambda+2 \mu}$ is the compressional wavenumber with the angular frequency $\omega$. We mention that the results obtained in the paper are same for the incidence of a shear plane wave $\boldsymbol{u}^{\mathrm{inc}}(\boldsymbol{x})=\boldsymbol{p} e^{\mathrm{i} \kappa_{2} \boldsymbol{q} \cdot \boldsymbol{x}}$, where $\boldsymbol{p}$ is a unit vector satisfying $\boldsymbol{p} \cdot \boldsymbol{q}=0$ and $\kappa_{2}=\omega / \sqrt{\mu}$ is the shear wavenumber, or a linear combination of the shear and compressional plane waves.

Denote the displacement of the scattered wave by $\boldsymbol{u}$, which satisfies the Navier wave equation

$$
\mu \Delta \boldsymbol{u}+(\lambda+\mu) \nabla \nabla \cdot \boldsymbol{u}+\omega^{2} \boldsymbol{u}=0 \quad \text { in } \Omega_{f} .
$$

Since the surface is assumed to be elastically rigid, we have

$$
\boldsymbol{u}=-\boldsymbol{u}^{\text {inc }} \text { on } S \text {. }
$$

In addition, the scattered wave $\boldsymbol{u}$ is assumed to satisfy the bounded outgoing wave condition as $x_{3} \rightarrow \infty$. Motivated by uniqueness, we seek the so-called quasi-periodic solutions of (2.1) and (2.2), i.e., $\boldsymbol{u}(\boldsymbol{x}) e^{-\mathrm{i} \boldsymbol{\alpha} \cdot \boldsymbol{r}}$ is a biperiodic function of $\boldsymbol{r}=\left(x_{1}, x_{2}\right)^{\top}$ with periods $\Lambda_{1}$ and $\Lambda_{2}$ in the $x_{1}$ and $x_{2}$ directions, respectively, where $\boldsymbol{\alpha}=\left(\alpha_{1}, \alpha_{2}\right)^{\top}, \alpha_{1}=\kappa_{1} \sin \theta_{1} \cos \theta_{2}, \alpha_{2}=\kappa_{1} \sin \theta_{1} \sin \theta_{2}$.

Define a quasi-periodic function space

$$
H_{\mathrm{qp}}^{1}(\Omega)=\left\{u \in H^{1}(\Omega): e^{\mathrm{i} \alpha_{1} \Lambda_{1}} u\left(0, x_{2}, x_{3}\right)=u\left(\Lambda_{1}, x_{2}, x_{3}\right), \quad e^{\mathrm{i} \alpha_{2} \Lambda_{2}} u\left(x_{1}, 0, x_{3}\right)=u\left(x_{1}, \Lambda_{2}, x_{3}\right)\right\}
$$

and its subspace

Let

$$
H_{S, \mathrm{qp}}^{1}(\Omega)=\left\{u \in H_{\mathrm{qp}}^{1}(\Omega): u=0 \text { on } S\right\} .
$$

$$
L_{\mathrm{qp}}^{2}\left(\Gamma_{h}\right)=\left\{u \in L^{2}\left(\Gamma_{h}\right): e^{\mathrm{i} \alpha_{1} \Lambda_{1}} u\left(0, x_{2}, h\right)=u\left(\Lambda_{1}, x_{2}, h\right), \quad e^{\mathrm{i} \alpha_{2} \Lambda_{2}} u\left(x_{1}, 0, h\right)=u\left(x_{1}, \Lambda_{2}, h\right)\right\} .
$$

For any $u \in L_{\mathrm{qp}}^{2}\left(\Gamma_{h}\right)$, it has the Fourier series expansion

$$
u(\boldsymbol{r}, h)=\sum_{n \in \mathbb{Z}^{2}} u_{n}(h) e^{\mathrm{i} \boldsymbol{\alpha}_{n} \cdot \boldsymbol{r}}, \quad u_{n}(h)=\frac{1}{\Lambda_{1} \Lambda_{2}} \int_{0}^{\Lambda_{1}} \int_{0}^{\Lambda_{2}} u(\boldsymbol{r}, h) e^{-\mathrm{i} \boldsymbol{\alpha}_{n} \cdot \boldsymbol{r}} \mathrm{d} \boldsymbol{r},
$$

where $n=\left(n_{1}, n_{2}\right)^{\top} \in \mathbb{Z}^{2}, \boldsymbol{\alpha}_{n}=\left(\alpha_{1 n}, \alpha_{2 n}\right)^{\top}, \alpha_{j n}=\alpha_{j}+2 \pi n_{j} / \Lambda_{j}, j=1,2$.

Define a trace function space $H^{s}\left(\Gamma_{h}\right), s \in \mathbb{R}^{+}$by

$$
H^{s}\left(\Gamma_{h}\right)=\left\{u \in L^{2}\left(\Gamma_{h}\right):\|u\|_{H^{s}\left(\Gamma_{h}\right)}<\infty\right\},
$$

where the norm is given by

$$
\|u\|_{H^{s}\left(\Gamma_{h}\right)}^{2}=\Lambda_{1} \Lambda_{2} \sum_{n \in \mathbb{Z}^{2}}\left(1+\left|\boldsymbol{\alpha}_{n}\right|^{2}\right)^{s}\left|u_{n}(h)\right|^{2} .
$$

It is clear that the dual space of $H^{s}\left(\Gamma_{h}\right)$ is $H^{-s}\left(\Gamma_{h}\right)$ with respect to the scalar product in $L^{2}\left(\Gamma_{h}\right)$ given by

$$
\langle u, v\rangle_{\Gamma_{h}}=\int_{\Gamma_{h}} u \bar{v} \mathrm{~d} s .
$$

Let $\boldsymbol{H}_{\mathrm{qp}}^{1}(\Omega), \boldsymbol{H}_{S, \mathrm{qp}}^{1}(\Omega)$ and $\boldsymbol{H}^{s}\left(\Gamma_{h}\right)$ be the Cartesian product spaces equipped with the corresponding 2norms of $H_{\mathrm{qp}}^{1}(\Omega), H_{S, \mathrm{qp}}^{1}(\Omega)$ and $H^{s}\left(\Gamma_{h}\right)$, respectively. Throughout the paper, the notation $a \lesssim b$ stands for $a \leqslant C b$, where $C$ is a positive constant whose value is not required but should be clear from the context. 


\section{The Boundary VAlue PROBlem}

In this section, we introduce the DtN operator to reduce the problem (2.1) and (2.2) into a boundary value problem in the bounded domain $\Omega$ and present the well-posedness of its variational formulation.

Consider the Helmholtz decomposition

$$
\boldsymbol{u}=\nabla \phi+\nabla \times \boldsymbol{\psi}, \quad \nabla \cdot \boldsymbol{\psi}=0 \quad \text { in } \Omega,
$$

where $\phi$ is a scalar potential function and $\boldsymbol{\psi}=\left(\psi_{1}, \psi_{2}, \psi_{3}\right)$ is a vector potential function. Substituting (3.1) into the Navier wave equation (2.1), we may verify that $\phi$ and $\boldsymbol{\psi}$ satisfy the following Helmholtz equation and the Maxwell equation, respectively:

$$
\Delta \phi+\kappa_{1}^{2} \phi=0, \quad \nabla \times(\nabla \times \boldsymbol{\psi})-\kappa_{2}^{2} \psi=0 .
$$

It is easy to verify from the Helmholtz decomposition (3.1) and the boundary condition (2.2) that $\phi$ and $\boldsymbol{\psi}$ satisfy the following coupled boundary conditions on $S$ :

$$
\partial_{\nu} \phi+(\nabla \times \boldsymbol{\psi}) \cdot \nu=-\boldsymbol{u}^{\mathrm{inc}} \cdot \nu, \quad(\nabla \times \boldsymbol{\psi}) \times \nu+\nabla \phi \times \nu=-\boldsymbol{u}^{\mathrm{inc}} \times \nu,
$$

where $\nu$ is the unit normal vector on $S$.

The potential functions $\phi$ and $\boldsymbol{\psi}$ are required to be quasi-periodic in $x_{1}$ and $x_{2}$ directions with periods $\Lambda_{1}$ and $\Lambda_{2}$. Hence they have the Fourier series expansions

$$
\phi(\boldsymbol{x})=\sum_{n \in \mathbb{Z}^{2}} \phi_{n}\left(x_{3}\right) e^{\mathrm{i} \boldsymbol{\alpha}_{n} \cdot \boldsymbol{r}}, \quad \boldsymbol{\psi}(\boldsymbol{x})=\sum_{n \in \mathbb{Z}^{2}} \boldsymbol{\psi}_{n}\left(x_{3}\right) e^{\mathrm{i} \boldsymbol{\alpha}_{n} \cdot \boldsymbol{r}}
$$

Plugging (3.4) into (3.2) and using the bounded outgoing wave condition, we have from a straight forward calculation that $\phi$ and $\boldsymbol{\psi}$ admit the following expansions for $x_{3}>h$ :

$$
\phi(\boldsymbol{x})=\sum_{n \in \mathbb{Z}^{2}} \phi_{n}(h) e^{\mathrm{i}\left(\boldsymbol{\alpha}_{n} \cdot \boldsymbol{r}+\beta_{1 n}\left(x_{3}-h\right)\right)}, \quad \boldsymbol{\psi}(\boldsymbol{x})=\sum_{n \in \mathbb{Z}^{2}} \boldsymbol{\psi}_{n}(h) e^{\mathrm{i}\left(\boldsymbol{\alpha}_{n} \cdot \boldsymbol{r}+\beta_{2 n}\left(x_{3}-h\right)\right)},
$$

where

$$
\beta_{j n}= \begin{cases}\left(\kappa_{j}^{2}-\left|\boldsymbol{\alpha}_{n}\right|^{2}\right)^{1 / 2} & \text { if }\left|\boldsymbol{\alpha}_{n}\right|<\kappa_{j} \\ \mathrm{i}\left(\left|\boldsymbol{\alpha}_{n}\right|^{2}-\kappa_{j}^{2}\right)^{1 / 2} & \text { if }\left|\boldsymbol{\alpha}_{n}\right|>\kappa_{j}\end{cases}
$$

It follows from (3.4) and (3.5) that

$$
\phi_{n}\left(x_{3}\right)=\phi_{n}(h) e^{\mathrm{i} \beta_{1 n}\left(x_{3}-h\right)}, \quad \psi_{j n}\left(x_{3}\right)=\psi_{j n}(h) e^{\mathrm{i} \beta_{2 n}\left(x_{3}-h\right)}, \quad j=1,2,3 .
$$

We observe from (3.5) and (3.6) that $\beta_{j n}$ is a pure imaginary number and thus $\phi_{n}$ and $\boldsymbol{\psi}_{n}$ are known as surface wave modes when $\left|\boldsymbol{\alpha}_{n}\right|>\kappa_{j}$.

Substituting (3.5) into (3.1), we obtain the representation of the scattered field $\boldsymbol{u}$ in terms of the Fourier coefficients of the potential functions $\phi$ and $\boldsymbol{\psi}$ :

$$
\boldsymbol{u}(\boldsymbol{x})=\mathrm{i} \sum_{n \in \mathbb{Z}^{2}}\left\{\left[\begin{array}{l}
\alpha_{1 n} \\
\alpha_{2 n} \\
\beta_{1 n}
\end{array}\right] \phi_{n}(h) e^{\mathrm{i} \beta_{1 n}\left(x_{3}-h\right)}+\left[\begin{array}{c}
\alpha_{2 n} \psi_{3 n}(h)-\beta_{2 n} \psi_{2 n}(h) \\
\beta_{2 n} \psi_{1 n}(h)-\alpha_{1 n} \psi_{3 n}(h) \\
\alpha_{1 n} \psi_{2 n}(h)-\alpha_{2 n} \psi_{1 n}(h)
\end{array}\right] e^{\mathrm{i} \beta_{2 n}\left(x_{3}-h\right)}\right\} e^{\mathrm{i} \boldsymbol{\alpha}_{n} \cdot \boldsymbol{r}} .
$$

Noting $\nabla \cdot \boldsymbol{\psi}=0$, we may represent conversely the coefficients of the potential functions of $\phi$ and $\boldsymbol{\psi}$ by the coefficients of the scattered field $\boldsymbol{u}=\left(u_{1}, u_{2}, u_{3}\right)^{\top}$ :

$$
\phi_{n}(h)=-\frac{\mathrm{i}}{\chi_{n}}\left(\alpha_{1 n} u_{1 n}(h)+\alpha_{2 n} u_{2 n}(h)+\beta_{2 n} u_{3 n}(h)\right),
$$




$$
\begin{aligned}
& \psi_{1 n}(h)=-\frac{\mathrm{i}}{\chi_{n}}\left(\frac{1}{\kappa_{2}^{2}} \alpha_{1 n} \alpha_{2 n}\left(\beta_{1 n}-\beta_{2 n}\right) u_{1 n}(h)-\alpha_{2 n} u_{3 n}(h)+\frac{1}{\kappa_{2}^{2}}\left[\alpha_{1 n}^{2} \beta_{2 n}+\alpha_{2 n}^{2} \beta_{1 n}+\beta_{1 n} \beta_{2 n}^{2}\right] u_{2 n}(h)\right), \\
& \psi_{2 n}(h)=-\frac{\mathrm{i}}{\chi_{n}}\left(-\frac{1}{\kappa_{2}^{2}}\left[\alpha_{1 n}^{2} \beta_{1 n}+\alpha_{2 n}^{2} \beta_{2 n}+\beta_{1 n} \beta_{2 n}^{2}\right] u_{1 n}(h)-\frac{1}{\kappa_{2}^{2}} \alpha_{1 n} \alpha_{2 n}\left(\beta_{1 n}-\beta_{2 n}\right) u_{2 n}(h)+\alpha_{1 n} u_{3 n}(h)\right) \\
& \psi_{3 n}(h)=-\frac{\mathrm{i}}{\kappa_{2}^{2}}\left(\alpha_{2 n} u_{1 n}(h)-\alpha_{1 n} u_{2 n}(h)\right),
\end{aligned}
$$

where $\chi_{n}=\left|\boldsymbol{\alpha}_{n}\right|^{2}+\beta_{1 n} \beta_{2 n}$. It is easy to verify that $\chi_{n} \neq 0$ for $n \in \mathbb{Z}^{2}$.

Define a differential operator

$$
D \boldsymbol{u}=\mu \partial_{x_{3}} \boldsymbol{u}+(\lambda+\mu)(\nabla \cdot \boldsymbol{u}) \boldsymbol{e}_{3} \quad \text { on } \Gamma_{h},
$$

where $\boldsymbol{e}_{3}=(0,0,1)^{\top}$. Substituting (3.8)-(3.12) into the differential operator $D$, we may deduce the DtN operator

$$
T \boldsymbol{u}=\sum_{n \in \mathbb{Z}^{2}} M_{n} \boldsymbol{u}_{n}(h) e^{\mathrm{i} \boldsymbol{\alpha}_{n} \cdot \boldsymbol{r}}
$$

where the matrix $M_{n}$ is defined as

$$
M_{n}=\frac{\mathrm{i} \mu}{\chi_{n}}\left[\begin{array}{ccc}
\alpha_{1 n}^{2} \beta_{12}^{(n)}+\beta_{2 n} \chi_{n} & \alpha_{1 n} \alpha_{2 n} \beta_{12}^{(n)} & \alpha_{1 n} \beta_{2 n} \beta_{12}^{(n)} \\
\alpha_{1 n} \alpha_{2 n} \beta_{12}^{(n)} & \alpha_{2 n}^{2} \beta_{12}^{(n)}+\beta_{2 n} \chi_{n} & \alpha_{2 n} \beta_{2 n} \beta_{12}^{(n)} \\
-\alpha_{1 n} \beta_{2 n} \beta_{12}^{(n)} & -\alpha_{2 n} \beta_{2 n} \beta_{12}^{(n)} & \kappa_{2}^{2} \beta_{2 n}
\end{array}\right]
$$

Here $\beta_{12}^{(n)}=\beta_{1 n}-\beta_{2 n}$. The details can be found in [28] for the derivation.

Based on the DtN operator (3.14), the scattering problem (2.1) and (2.2) can be equivalently reduced to the following boundary value problem:

$$
\begin{cases}\mu \Delta \boldsymbol{u}+(\lambda+\mu) \nabla \nabla \cdot \boldsymbol{u}+\omega^{2} \boldsymbol{u}=0 & \text { in } \Omega \\ D \boldsymbol{u}=T \boldsymbol{u} & \text { on } \Gamma_{h} \\ \boldsymbol{u}=-\boldsymbol{u}^{\text {inc }} & \text { on } S .\end{cases}
$$

The variational problem of (3.16) is to find $\boldsymbol{u} \in \boldsymbol{H}_{\mathrm{qp}}^{1}(\Omega)$ with $\boldsymbol{u}=-\boldsymbol{u}^{\mathrm{inc}}$ on $S$ such that

$$
a(\boldsymbol{u}, \boldsymbol{v})=0 \quad \forall \boldsymbol{v} \in \boldsymbol{H}_{S, \mathrm{qp}}^{1}(\Omega)
$$

where the sesquilinear form $a: H_{\mathrm{qp}}^{1}(\Omega) \times H_{\mathrm{qp}}^{1}(\Omega) \rightarrow \mathbb{C}$ is

$$
a(\boldsymbol{u}, \boldsymbol{v})=\mu \int_{\Omega} \nabla \boldsymbol{u}: \nabla \overline{\boldsymbol{v}} \mathrm{d} \boldsymbol{x}+(\lambda+\mu) \int_{\Omega}(\nabla \cdot \boldsymbol{u})(\nabla \cdot \overline{\boldsymbol{v}}) \mathrm{d} \boldsymbol{x}-\omega^{2} \int_{\Omega} \boldsymbol{u} \cdot \overline{\boldsymbol{v}} \mathrm{d} \boldsymbol{x}-\int_{\Gamma_{h}} T \boldsymbol{u} \cdot \overline{\boldsymbol{v}} \mathrm{d} s .
$$

Here $A: B=\operatorname{tr}\left(A B^{\top}\right)$ is the Frobenius inner product of two square matrices $A$ and $B$.

The well-posedness of the variational problem (3.17) was discussed in [20]. It was shown that the variational problem has a unique weak solution for all incident waves. Thus, the solution satisfies the estimates

$$
\|\boldsymbol{u}\|_{\boldsymbol{H}^{1}(\Omega)} \lesssim\left\|\boldsymbol{u}^{\mathrm{inc}}\right\|_{\boldsymbol{H}^{1 / 2}(S)} \lesssim\left\|\boldsymbol{u}^{\mathrm{inc}}\right\|_{\boldsymbol{H}^{1}(\Omega)} .
$$

By the general theory of Babuška and Aziz [3], there exists a $\gamma>0$ such that the following inf-sup condition holds:

$$
\sup _{0 \neq \boldsymbol{v} \in \boldsymbol{H}_{\mathrm{qp}}^{1}(\Omega)} \frac{|a(\boldsymbol{u}, \boldsymbol{v})|}{\|\boldsymbol{v}\|_{\boldsymbol{H}^{1}(\Omega)}} \geqslant \gamma\|\boldsymbol{u}\|_{\boldsymbol{H}^{1}(\Omega)} \quad \forall \boldsymbol{u} \in \boldsymbol{H}_{\mathrm{qp}}^{1}(\Omega) .
$$




\section{The Finite ElEMEnt APproximation}

Since the non-local DtN operator (3.14) is given as an infinite series, in practice, it needs to be truncated into a sum of finitely many terms

$$
T_{N} \boldsymbol{u}=\sum_{\left|n_{1}\right|,\left|n_{2}\right| \leqslant N} M_{n} \boldsymbol{u}_{n}(h) e^{\mathrm{i} \boldsymbol{\alpha}_{n} \cdot \boldsymbol{r}} \quad \text { on } \Gamma_{h},
$$

where $N>0$ is a sufficiently large integer. Using (4.1), we arrive at the truncated finite element approximation: find $\boldsymbol{u}_{N} \in \boldsymbol{H}_{q p}^{1}(\Omega)$ with $\boldsymbol{u}_{N}=-\boldsymbol{u}^{\text {inc }}$ on $S$ such that

$$
a_{N}\left(\boldsymbol{u}_{N}, \boldsymbol{v}\right)=0 \quad \forall \boldsymbol{v} \in \boldsymbol{H}_{S, q p}^{1},
$$

where the sesquilinear form $a_{N}: \boldsymbol{H}^{1}(\Omega) \times \boldsymbol{H}^{1}(\Omega) \rightarrow \mathbb{C}$ is

$$
a_{N}(\boldsymbol{u}, \boldsymbol{v})=\mu \int_{\Omega} \nabla \boldsymbol{u}: \nabla \overline{\boldsymbol{v}} \mathrm{d} \boldsymbol{x}+(\lambda+\mu) \int_{\Omega}(\nabla \cdot \boldsymbol{u})(\nabla \cdot \overline{\boldsymbol{v}}) \mathrm{d} \boldsymbol{x}-\omega^{2} \int_{\Omega} \boldsymbol{u} \cdot \overline{\boldsymbol{v}} \mathrm{d} \boldsymbol{x}-\int_{\Gamma_{h}} T_{N} \boldsymbol{u} \cdot \overline{\boldsymbol{v}} \mathrm{d} s .
$$

Let $\mathcal{M}_{h}$ be a regular tetrahedral mesh of the domain $\Omega$, where $h$ denotes the maximum diameter of all the elements in $\mathcal{M}_{h}$. To handle the quasi-periodic solution, we assume that the mesh is periodic in both $x_{1}$ and $x_{2}$ directions, i.e., the surface meshes on the planes $x_{1}=0$ and $x_{2}=0$ coincide with the surface meshes on the planes $x_{1}=\Lambda_{1}$ and $x_{2}=\Lambda_{2}$, respectively. We also assume for simplicity that $S$ is polygonal to keep from using the isoparametric finite element space and deriving the approximation error of the boundary $S$ in order to avoid being distracted from the main focus of the a posteriori error analysis.

Let $V_{h} \subset \boldsymbol{H}_{\mathrm{qp}}^{1}(\Omega)$ be a conforming finite element space, i.e.

$$
V_{h}=\left\{\boldsymbol{v} \in C_{\mathrm{qp}}(\Omega)^{3}:\left.\boldsymbol{v}\right|_{K} \in P_{m}(K)^{3} \forall K \in \mathcal{M}_{h}\right\},
$$

where $C_{\mathrm{qp}}(\Omega)$ is the set of all continuous functions satisfying the quasi-periodic boundary condition, $m$ is a positive integer, and $P_{m}$ denotes the set of all polynomials with degree no more than $m$.

Then the finite element approximation to the variational problem (4.2) is to find $\boldsymbol{u}_{N}^{h} \in V_{h}$ such that $\boldsymbol{u}_{N}^{h}=-\boldsymbol{g}^{h}$ on $S$ and satisfies the variational problem

$$
a_{N}\left(\boldsymbol{u}_{N}^{h}, \boldsymbol{v}^{h}\right)=0 \quad \forall \boldsymbol{v}^{h} \in V_{h, S},
$$

where $V_{h, S}=\left\{\boldsymbol{v} \in V_{h}: \boldsymbol{v}=0\right.$ on $\left.S\right\}$ and $\boldsymbol{g}^{h}$ is the finite element approximation of $\boldsymbol{u}^{\text {inc }}$.

Following the argument in [24], it can be shown that for sufficiently large $N$ the variational problem (4.2) is well-posed. And for sufficient large $N$ and small enough $h$, the discrete inf-sup condition of the sesquilinear form $a_{N}$ can be established by following the approach in [37]. Based on the general theory in [3], it can be shown that the discrete variational problem (4.3) has a unique solution $\boldsymbol{u}_{N}^{h} \in V_{h}$. The details are omitted for brevity since our focus is on the a posteriori error estimate.

\section{THE A POSTERIORI ERROR ANALYSIS}

For any tetrahedral element $K \in \mathcal{M}_{h}$, denote by $h_{K}$ its diameter. Define the operator residual in $K$ as

$$
R_{K} \boldsymbol{u}_{N}^{h}=\left.\left(\mu \Delta \boldsymbol{u}_{N}^{h}+(\lambda+\mu) \nabla \nabla \cdot \boldsymbol{u}_{N}^{h}+\omega^{2} \boldsymbol{u}_{N}^{h}\right)\right|_{K} .
$$

Let $\mathcal{F}_{h}$ be the set of all the faces on $\mathcal{M}_{h}$. Given any interior face $F \in \mathcal{F}_{h}$, which is the common face of tetrahedral element $K_{1}$ and $K_{2}$, we define the jump residual across $F$ as

$$
J_{F} \boldsymbol{u}_{N}^{h}=\left[\left[\left.\mu \nabla \boldsymbol{u}_{N}^{h}\right|_{K_{j}} \cdot \nu_{1}+(\lambda+\mu)\left(\left.\nabla \cdot \boldsymbol{u}_{N}^{h}\right|_{K_{j}}\right) \nu_{1}\right]\right]
$$


where $\nu_{j}, j=1,2$ is the unit outward normal vector on the face of $K_{j}$. For any boundary face $F \in \mathcal{F}_{h} \cap \Gamma_{h}$, define the jump residual as

$$
J_{F} \boldsymbol{u}_{N}^{h}=2\left(T_{N} \boldsymbol{u}_{N}^{h}-D \boldsymbol{u}_{N}^{h}\right) .
$$

Denote the four lateral boundary surfaces by

$$
\begin{aligned}
& \Gamma_{10}=\left\{\boldsymbol{x} \in \mathbb{R}^{3}: x_{1}=0,0<x_{2}<\Lambda_{2}, f\left(0, x_{2}\right)<x_{3}<h\right\}, \\
& \Gamma_{11}=\left\{\boldsymbol{x} \in \mathbb{R}^{3}: x_{1}=\Lambda_{1}, 0<x_{2}<\Lambda_{2}, f\left(\Lambda_{1}, x_{2}\right)<x_{3}<h\right\}, \\
& \Gamma_{20}=\left\{\boldsymbol{x} \in \mathbb{R}^{3}: 0<x_{1}<\Lambda_{1}, x_{2}=0, f\left(x_{1}, 0\right)<x_{3}<h\right\}, \\
& \Gamma_{21}=\left\{\boldsymbol{x} \in \mathbb{R}^{3}: 0<x_{1}<\Lambda_{1}, x_{2}=\Lambda_{2}, f\left(x_{1}, \Lambda_{2}\right)<x_{3}<h\right\} .
\end{aligned}
$$

For any boundary face $F \in \Gamma_{10}$ and the corresponding face $F^{\prime} \in \Gamma_{11}$, if $F \in K_{1}$ and $F^{\prime} \in K_{2}$, then the jump residual is defined as

$$
\begin{aligned}
J_{F}^{(1)} \boldsymbol{u}_{N}^{h} & =\left[\left.\mu \partial_{x_{1}} \boldsymbol{u}_{N}^{h}\right|_{K_{1}}+(\lambda+\mu)\left(\left.\nabla \cdot \boldsymbol{u}_{N}^{h}\right|_{K_{1}}\right) \boldsymbol{e}_{1}\right]-e^{-\mathrm{i} \alpha_{1} \Lambda_{1}}\left[\left.\mu \partial_{x_{1}} \boldsymbol{u}_{N}^{h}\right|_{K_{2}}+(\lambda+\mu)\left(\left.\nabla \cdot \boldsymbol{u}_{N}^{h}\right|_{K_{2}}\right) \boldsymbol{e}_{1}\right], \\
J_{F^{\prime}}^{(1)} \boldsymbol{u}_{N}^{h} & =e^{\mathrm{i} \alpha_{1} \Lambda_{1}}\left[\left.\mu \partial_{x_{1}} \boldsymbol{u}_{N}^{h}\right|_{K_{1}}+(\lambda+\mu)\left(\left.\nabla \cdot \boldsymbol{u}_{N}^{h}\right|_{K_{1}}\right) \boldsymbol{e}_{1}\right]-\left[\left.\mu \partial_{x_{1}} \boldsymbol{u}_{N}^{h}\right|_{K_{2}}+(\lambda+\mu)\left(\left.\nabla \cdot \boldsymbol{u}_{N}^{h}\right|_{K_{2}}\right) \boldsymbol{e}_{1}\right],
\end{aligned}
$$

where $\boldsymbol{e}_{1}=(1,0,0)^{\top}$. Similarly, for any face $F \in \Gamma_{20}$ and its corresponding face $F^{\prime} \in \Gamma_{21}$, the jump residual is defined as

$$
\begin{aligned}
J_{F}^{(2)} \boldsymbol{u}_{N}^{h} & =\left[\left.\mu \partial_{x_{2}} \boldsymbol{u}_{N}^{h}\right|_{K_{1}}+(\lambda+\mu)\left(\left.\nabla \cdot \boldsymbol{u}_{N}^{h}\right|_{K_{1}}\right) \boldsymbol{e}_{2}\right]-e^{-\mathrm{i} \alpha_{2} \Lambda_{2}}\left[\left.\mu \partial_{x_{2}} \boldsymbol{u}_{N}^{h}\right|_{K_{2}}+(\lambda+\mu)\left(\left.\nabla \cdot \boldsymbol{u}_{N}^{h}\right|_{K_{2}}\right) \boldsymbol{e}_{2}\right], \\
J_{F^{\prime}}^{(2)} \boldsymbol{u}_{N}^{h} & =e^{\mathrm{i} \alpha_{2} \Lambda_{2}}\left[\left.\mu \partial_{x_{2}} \boldsymbol{u}_{N}^{h}\right|_{K_{1}}+(\lambda+\mu)\left(\left.\nabla \cdot \boldsymbol{u}_{N}^{h}\right|_{K_{1}}\right) \boldsymbol{e}_{2}\right]-\left[\left.\mu \partial_{x_{2}} \boldsymbol{u}\right|_{K_{2}}+(\lambda+\mu)\left(\left.\nabla \cdot \boldsymbol{u}_{N}^{h}\right|_{K_{2}}\right) \boldsymbol{e}_{2}\right],
\end{aligned}
$$

where $\boldsymbol{e}_{2}=(0,1,0)^{\top}$.

For any tetrahedral element $K \in \mathcal{M}_{h}$, denote by $\eta_{K}$ the local error estimator as follows:

$$
\eta_{K}^{2}=h_{K}^{2}\left\|R_{K} \boldsymbol{u}_{N}^{h}\right\|_{\boldsymbol{L}^{2}(K)}^{2}+h_{K} \sum_{F \subset \partial K}\left(\left\|J_{F}^{(1)} \boldsymbol{u}_{N}^{h}\right\|_{\boldsymbol{L}^{2}(F)}^{2}+\left\|J_{F}^{(2)} \boldsymbol{u}_{N}^{h}\right\|_{\boldsymbol{L}^{2}(F)}^{2}\right) .
$$

For convenience, we introduce a weighted $\boldsymbol{H}^{1}(\Omega)$ norm

$$
\|\boldsymbol{u}\|_{\boldsymbol{H}^{1}(\Omega)}^{2}=\mu \int_{\Omega}|\nabla \boldsymbol{u}|^{2} \mathrm{~d} \boldsymbol{x}+(\lambda+\mu) \int_{\Omega}|\nabla \cdot \boldsymbol{u}|^{2} \mathrm{~d} \boldsymbol{x}+\omega^{2} \int_{\Omega}|\boldsymbol{u}|^{2} \mathrm{~d} \boldsymbol{x} .
$$

Since $\mu$ and $\lambda+\mu$ are positive, it is easy to check that

$$
\min \left(\mu, \omega^{2}\right)\|\boldsymbol{u}\|_{\boldsymbol{H}^{1}(\Omega)}^{2} \leqslant\|\boldsymbol{u}\|_{\boldsymbol{H}^{1}(\Omega)}^{2} \leqslant \max \left(2 \lambda+3 \mu, \omega^{2}\right)\|\boldsymbol{u}\|_{\boldsymbol{H}^{1}(\Omega)}^{2} \quad \forall \boldsymbol{u} \in \boldsymbol{H}^{1}(\Omega),
$$

which implies that the weighted $\boldsymbol{H}^{1}(\Omega)$ norm (5.1) is equivalent to the standard $\boldsymbol{H}^{1}(\Omega)$ norm.

The following theorem is the main result of the paper. It presents the a posteriori error estimate between the solutions of the original scattering problem (3.17) and the truncated finite element approximation (4.3).

Theorem 5.1. Let $\boldsymbol{u}$ and $\boldsymbol{u}_{N}^{h}$ be the solutions of the variational problems (3.17) and (4.3), respectively. Then for any $\hat{h}$ such that $\max _{\boldsymbol{r} \in \mathbb{R}^{2}} f(\boldsymbol{r})<\hat{h}<h$ and for sufficiently large $N$, the following a posteriori error estimate holds:

$$
\left\|\boldsymbol{u}-\boldsymbol{u}_{N}^{h}\right\|_{\boldsymbol{H}^{1}(\Omega)} \lesssim\left(\sum_{K \in \mathcal{M}_{h}} \eta_{K}^{2}\right)^{1 / 2}+\left\|\boldsymbol{u}^{\mathrm{inc}}-\boldsymbol{g}^{h}\right\|_{\boldsymbol{H}^{1 / 2}(S)}+\max _{|n|_{\min }>N}\left(|n|_{\max } e^{-\left|\beta_{2 n}\right|(h-\hat{h})}\right)\left\|\boldsymbol{u}^{\mathrm{inc}}\right\|_{\boldsymbol{H}^{1}(\Omega)},
$$

where $|n|_{\min }=\min \left(\left|n_{1}\right|,\left|n_{2}\right|\right)$ and $|n|_{\max }=\max \left(\left|n_{1}\right|,\left|n_{2}\right|\right)$. 
The a posteriori error (5.2) contains three parts: the first two parts arise from the finite element discretization error; the third part accounts for the truncation error of the DtN operator. Since $\hat{h}<h$, the latter is almost exponentially decaying. Hence the DtN truncated error can be controlled to be small enough so that it does not contaminate the finite element discretization error.

To prove Theorem 5.1, let us begin with the following trace result in $\boldsymbol{H}_{\mathrm{qp}}^{1}(\Omega)$. The proof can be found in Lemma 3.3 of [28].

Lemma 5.2. Let $a=\min _{\boldsymbol{r} \in \mathbb{R}^{2}} f(\boldsymbol{r})$. Then for any $\boldsymbol{u} \in \boldsymbol{H}_{\mathrm{qp}}^{1}(\Omega)$ the following estimate holds:

$$
\|\boldsymbol{u}\|_{\boldsymbol{H}^{1 / 2}\left(\Gamma_{h}\right)} \leqslant C\|\boldsymbol{u}\|_{\boldsymbol{H}^{1}(\Omega)},
$$

where $C=\left(1+(h-a)^{-1}\right)^{1 / 2}$.

Denote by $\boldsymbol{\xi}=\boldsymbol{u}-\boldsymbol{u}_{N}^{h}$ the error between the solutions of (3.17) and (4.3), then a simple calculation yields

$$
\begin{aligned}
\|\|_{H^{1}(\Omega)}^{2} & =\mu \int_{\Omega} \nabla \boldsymbol{\xi}: \nabla \overline{\boldsymbol{\xi}} \mathrm{d} \boldsymbol{x}+(\lambda+\mu) \int_{\Omega}(\nabla \cdot \boldsymbol{\xi})(\nabla \cdot \overline{\boldsymbol{\xi}}) \mathrm{d} \boldsymbol{x}+\omega^{2} \int_{\Omega} \boldsymbol{\xi} \cdot \overline{\boldsymbol{\xi}} \mathrm{d} \boldsymbol{x} \\
& =\Re a(\boldsymbol{\xi}, \boldsymbol{\xi})+2 \omega^{2} \int_{\Omega} \boldsymbol{\xi} \cdot \overline{\boldsymbol{\xi}} \mathrm{d} \boldsymbol{x}+\Re \int_{\Gamma_{h}} T \boldsymbol{\xi} \cdot \overline{\boldsymbol{\xi}} \mathrm{d} s \\
& =\Re a(\boldsymbol{\xi}, \boldsymbol{\xi})+\Re \int_{\Gamma_{h}}\left(T-T_{N}\right) \boldsymbol{\xi} \cdot \overline{\boldsymbol{\xi}} \mathrm{d} s+2 \omega^{2} \int_{\Omega} \boldsymbol{\xi} \cdot \overline{\boldsymbol{\xi}} \mathrm{d} \boldsymbol{x}+\Re \int_{\Gamma_{h}} T_{N} \boldsymbol{\xi} \cdot \overline{\boldsymbol{\xi}} \mathrm{d} s .
\end{aligned}
$$

Due to the equivalence of the weighted norm $\|\cdot\|_{\boldsymbol{H}^{1}(\Omega)}$ to the standard norm $\|\cdot\|_{\boldsymbol{H}^{1}(\Omega)}$, it suffices to estimate the four terms on the right hand side of (5.3) one by one. The estimates of the first two terms are given in Lemmas 5.3 and 5.4.

Lemma 5.3. Let $\boldsymbol{u} \in \boldsymbol{H}_{\mathrm{qp}}^{1}(\Omega)$ be the solution of variational problem (3.17). For any $\boldsymbol{v} \in \boldsymbol{H}_{\mathrm{qp}}^{1}(\Omega)$ and a sufficiently large $N$, the following estimate holds:

$$
\left|\int_{\Gamma_{h}}\left(T-T_{N}\right) \boldsymbol{u} \cdot \overline{\boldsymbol{v}} \mathrm{d} s\right| \lesssim \max _{|n|_{\min }>N}\left(|n|_{\max } e^{-\left|\beta_{2 n}\right|(h-\hat{h})}\right)\left\|\boldsymbol{u}^{\mathrm{inc}}\right\|_{\boldsymbol{H}^{1}(\Omega)}\|\boldsymbol{v}\|_{\boldsymbol{H}^{1}(\Omega)} .
$$

Proof. It follows from (3.7) that we have

$$
\phi_{n}(h)=\phi_{n}(\hat{h}) e^{\mathrm{i} \beta_{1 n}(h-\hat{h})}, \quad \psi_{j n}(h)=\psi_{j n}(\hat{h}) e^{\mathrm{i} \beta_{2 n}(h-\hat{h})}, \quad j=1,2,3 .
$$

Substituting (5.4) into (3.8), we obtain the Fourier coefficients of $\boldsymbol{u}$ at $x_{3}=h$ in terms of the Fourier coefficients of $\phi$ and $\boldsymbol{\psi}$ at $x_{3}=\hat{h}$ :

$$
\begin{aligned}
{\left[\begin{array}{c}
u_{1 n}(h) \\
u_{2 n}(h) \\
u_{3 n}(h) \\
0
\end{array}\right] } & =\mathrm{i}\left[\begin{array}{cccc}
\alpha_{1 n} & 0 & -\beta_{2 n} & \alpha_{2 n} \\
\alpha_{2 n} & \beta_{2 n} & 0 & -\alpha_{1 n} \\
\beta_{1 n} & -\alpha_{2 n} & \alpha_{1 n} & 0 \\
0 & \alpha_{1 n} & \alpha_{2 n} & \beta_{2 n}
\end{array}\right]\left[\begin{array}{c}
\phi_{n}(h) \\
\psi_{1 n}(h) \\
\psi_{2 n}(h) \\
\psi_{3 n}(h)
\end{array}\right] \\
& =\mathrm{i}\left[\begin{array}{cccc}
\alpha_{1 n} & 0 & -\beta_{2 n} & \alpha_{2 n} \\
\alpha_{2 n} & \beta_{2 n} & 0 & -\alpha_{1 n} \\
\beta_{1 n}-\alpha_{2 n} & \alpha_{1 n} & 0 \\
0 & \alpha_{1 n} & \alpha_{2 n} & \beta_{2 n}
\end{array}\right] \operatorname{diag}\left(\left[\begin{array}{c}
e^{\mathrm{i} \beta_{1 n}(h-\hat{h})} \\
e^{\mathrm{i} \beta_{2 n}(h-\hat{h})} \\
e^{\mathrm{i} \beta_{2 n}(h-\hat{h})} \\
e^{\mathrm{i} \beta_{2 n}(h-\hat{h})}
\end{array}\right]\right)\left[\begin{array}{c}
\phi_{n}(\hat{h}) \\
\psi_{1 n}(\hat{h}) \\
\psi_{2 n}(\hat{h}) \\
\psi_{3 n}(\hat{h})
\end{array}\right]
\end{aligned}
$$




$$
:=\mathrm{i} A_{n}\left(\phi_{n}(\hat{h}), \psi_{1 n}(\hat{h}), \psi_{2 n}(\hat{h}), \psi_{3 n}(\hat{h})\right)^{\top} \text {. }
$$

Replacing $h$ by $\hat{h}$ in (3.9)-(3.12), we may equivalently have the matrix form

$$
\left[\phi_{n}(\hat{h}), \boldsymbol{\psi}_{n}(\hat{h})\right]=-\frac{\mathrm{i}}{\chi_{n}} B_{n} \boldsymbol{u}_{n}(\hat{h}),
$$

where the entries of the $4 \times 3$ matrix $B_{n}$ are

$$
\begin{aligned}
& B_{11}^{(n)}=\alpha_{1 n}, \quad B_{12}^{(n)}=\alpha_{2 n}, \quad B_{13}^{(n)}=\beta_{2 n}, \quad B_{23}^{(n)}=-\alpha_{2 n}, \quad B_{33}^{(n)}=\alpha_{1 n}, \quad B_{43}^{(n)}=0, \\
& B_{21}^{(n)}=-B_{32}^{(n)}=\frac{1}{\kappa_{2}^{2}} \alpha_{1 n} \alpha_{2 n}\left(\beta_{1 n}-\beta_{2 n}\right), \quad B_{41}^{(n)}=\frac{1}{\kappa_{2}^{2}} \alpha_{2 n} \chi_{n}, \quad B_{42}^{(n)}=-\frac{1}{\kappa_{2}^{2}} \alpha_{1 n} \chi_{n}, \\
& B_{22}^{(n)}=\frac{1}{\kappa_{2}^{2}}\left(\alpha_{1 n}^{2} \beta_{2 n}+\alpha_{2 n}^{2} \beta_{1 n}+\beta_{1 n} \beta_{2 n}^{2}\right), \quad B_{31}^{(n)}=-\frac{1}{\kappa_{2}^{2}}\left(\alpha_{1 n}^{2} \beta_{1 n}+\alpha_{2 n}^{2} \beta_{2 n}+\beta_{1 n} \beta_{2 n}^{2}\right) .
\end{aligned}
$$

Plugging (5.5) into (5.6) yields

$$
\left[\begin{array}{l}
u_{1 n}(h) \\
u_{2 n}(h) \\
u_{3 n}(h)
\end{array}\right]=\left.\frac{1}{\chi_{n}}\left(A_{n} B_{n}\right)\right|_{3 \times 3}\left[\begin{array}{l}
u_{1 n}(\hat{h}) \\
u_{2 n}(\hat{h}) \\
u_{3 n}(\hat{h})
\end{array}\right]:=\frac{1}{\chi_{n}} P_{n}\left[\begin{array}{l}
u_{1 n}(\hat{h}) \\
u_{2 n}(\hat{h}) \\
u_{3 n}(\hat{h})
\end{array}\right],
$$

where $\left.\left(A_{n} B_{n}\right)\right|_{3 \times 3}$ is the leading principal submatrix of order 3 of the matrix $A_{n} B_{n}$. A straight forward computation yields that

$$
\begin{aligned}
& P_{11}^{(n)}=\alpha_{1 n}^{2} e^{\mathrm{i} \beta_{1 n}(h-\hat{h})}+\frac{1}{\kappa_{2}^{2}} \alpha_{2 n}^{2} \chi_{n} e^{\mathrm{i} \beta_{2 n}(h-\hat{h})}+\frac{1}{\kappa_{2}^{2}}\left(\alpha_{1 n}^{2} \beta_{1 n}+\alpha_{2 n}^{2} \beta_{2 n}+\beta_{1 n} \beta_{2 n}^{2}\right) \beta_{2 n} e^{\mathrm{i} \beta_{2 n}(h-\hat{h})} \\
& P_{12}^{(n)}=\alpha_{1 n} \alpha_{2 n} e^{\mathrm{i} \beta_{1 n}(h-\hat{h})}+\frac{1}{\kappa_{2}^{2}} \alpha_{1 n} \alpha_{2 n}\left(\beta_{1 n}-\beta_{2 n}\right) \beta_{2 n} e^{\mathrm{i} \beta_{2 n}(h-\hat{h})}-\frac{1}{\kappa_{2}^{2}} \alpha_{1 n} \alpha_{2 n} \chi_{n} e^{\mathrm{i} \beta_{2 n}(h-\hat{h})}, \\
& P_{13}^{(n)}=\alpha_{1 n} \beta_{2 n}\left(e^{\mathrm{i} \beta_{1 n}(h-\hat{h})}-e^{\mathrm{i} \beta_{2 n}(h-\hat{h})}\right) \\
& P_{21}^{(n)}=\alpha_{1 n} \alpha_{2 n} e^{\mathrm{i} \beta_{1 n}(h-\hat{h})}+\frac{1}{\kappa_{2}^{2}} \alpha_{1 n} \alpha_{2 n}\left(\beta_{1 n}-\beta_{2 n}\right) \beta_{2 n} e^{\mathrm{i} \beta_{2 n}(h-\hat{h})}-\frac{1}{\kappa_{2}^{2}} \alpha_{1 n} \alpha_{2 n} \chi_{n} e^{\mathrm{i} \beta_{2 n}(h-\hat{h})}, \\
& P_{22}^{(n)}=\alpha_{2 n}^{2} e^{\mathrm{i} \beta_{1 n}(h-\hat{h})}+\frac{1}{\kappa_{2}^{2}} \alpha_{1 n}^{2} \chi_{n} e^{\mathrm{i} \beta_{2 n}(h-\hat{h})}+\frac{1}{\kappa_{2}^{2}}\left(\alpha_{1 n}^{2} \beta_{2 n}+\alpha_{2 n}^{2} \beta_{1 n}+\beta_{1 n} \beta_{2 n}^{2}\right) \beta_{2 n} e^{\mathrm{i} \beta_{2 n}(h-\hat{h})}, \\
& P_{23}^{(n)}=\alpha_{2 n} \beta_{2 n}\left(e^{\mathrm{i} \beta_{1 n}(h-\hat{h})}-e^{\mathrm{i} \beta_{2 n}(h-\hat{h})}\right), \\
& P_{31}^{(n)}=\alpha_{1 n} \beta_{1 n} e^{\mathrm{i} \beta_{1 n}(h-\hat{h})}-\frac{1}{\kappa_{2}^{2}} \alpha_{1 n} \alpha_{2 n}^{2}\left(\beta_{1 n}-\beta_{2 n}\right) e^{\mathrm{i} \beta_{2 n}(h-\hat{h})}-\frac{1}{\kappa_{2}^{2}}\left(\alpha_{1 n}^{2} \beta_{1 n}+\alpha_{2 n}^{2} \beta_{2 n}+\beta_{1 n} \beta_{2 n}^{2}\right) \alpha_{1 n} e^{\mathrm{i} \beta_{2 n}(h-\hat{h})}, \\
& P_{32}^{(n)}=\alpha_{2 n} \beta_{1 n} e^{\mathrm{i} \beta_{1 n}(h-\hat{h})}-\frac{1}{\kappa_{2}^{2}} \alpha_{2 n} \alpha_{1 n}^{2}\left(\beta_{1 n}-\beta_{2 n}\right) e^{\mathrm{i} \beta_{2 n}(h-\hat{h})}-\frac{1}{\kappa_{2}^{2}}\left(\alpha_{1 n}^{2} \beta_{2 n}+\alpha_{2 n}^{2} \beta_{1 n}+\beta_{1 n} \beta_{2 n}^{2}\right) \alpha_{2 n} e^{\mathrm{i} \beta_{2 n}(h-\hat{h})}, \\
& P_{33}^{(n)}=\beta_{1 n} \beta_{2 n} e^{\mathrm{i} \beta_{1 n}(h-\hat{h})}+\left(\alpha_{1 n}^{2}+\alpha_{2 n}^{2}\right) e^{\mathrm{i} \beta_{2 n}(h-\hat{h})} .
\end{aligned}
$$

When $\left|\boldsymbol{\alpha}_{n}\right|^{2}=\alpha_{1 n}^{2}+\alpha_{2 n}^{2}>\kappa_{2}^{2}$, it follows from (3.6) that both $\beta_{1 n}$ and $\beta_{2 n}$ are pure imaginary numbers. We may easily show

$$
\chi_{n}=\left|\boldsymbol{\alpha}_{n}\right|^{2}-\left(\left|\boldsymbol{\alpha}_{n}\right|^{2}-\kappa_{1}^{2}\right)^{1 / 2}\left(\left|\boldsymbol{\alpha}_{n}\right|^{2}-\kappa_{2}^{2}\right)^{1 / 2}<\kappa_{1}^{2}+\kappa_{2}^{2}
$$


and

$$
\mathrm{i}\left(\beta_{2 n}-\beta_{1 n}\right)=\left(\left|\boldsymbol{\alpha}_{n}\right|^{2}-\kappa_{1}^{2}\right)^{1 / 2}-\left(\left|\boldsymbol{\alpha}_{n}\right|^{2}-\kappa_{2}^{2}\right)^{1 / 2}<\frac{\kappa_{2}^{2}-\kappa_{1}^{2}}{2\left(\left|\boldsymbol{\alpha}_{n}\right|^{2}-\kappa_{2}^{2}\right)^{1 / 2}} .
$$

Plugging (3.6) and (5.8), (5.9) into $P_{n}$, we obtain

$$
\begin{aligned}
P_{11}^{(n)} & =\alpha_{1 n}^{2} e^{\mathrm{i} \beta_{1 n}(h-\hat{h})}+\frac{1}{\kappa_{2}^{2}} e^{\mathrm{i} \beta_{2 n}(h-\hat{h})}\left\{\left|\boldsymbol{\alpha}_{n}\right|^{2} \beta_{1 n} \beta_{2 n}-\alpha_{2 n}^{2}\left(\left|\boldsymbol{\alpha}_{n}\right|^{2}-\kappa_{2}^{2}\right)-\beta_{1 n} \beta_{2 n}\left(\left|\boldsymbol{\alpha}_{n}\right|^{2}-\kappa_{2}^{2}\right)+\alpha_{2 n}^{2}\left|\boldsymbol{\alpha}_{n}\right|^{2}\right\} \\
& =\alpha_{1 n}^{2} e^{\mathrm{i} \beta_{1 n}(h-\hat{h})}+e^{\mathrm{i} \beta_{2 n}(h-\hat{h})}\left(\alpha_{2 n}^{2}+\beta_{1 n} \beta_{2 n}\right) \\
& =\alpha_{1 n}^{2}\left(e^{\mathrm{i} \beta_{1 n}(h-\hat{h})}-e^{\mathrm{i} \beta_{2 n}(h-\hat{h})}\right)+e^{\mathrm{i} \beta_{2 n}(h-\hat{h})} \chi_{n},
\end{aligned}
$$

which gives

$$
\left|P_{11}^{(n)}\right| \lesssim|n|_{\max } e^{-\left|\beta_{2 n}\right|(h-\hat{h})}
$$

Similarly, we may show that all the entries of the matrix $P_{n}$ have the estimates

$$
\left|P_{i j}^{(n)}\right| \lesssim|n|_{\max } e^{-\left|\beta_{2 n}\right|(h-\hat{h})}, \quad i, j=1,2,3 .
$$

Substituting (5.8) and (5.10) into (5.7) gives

$$
\left|\boldsymbol{u}_{n}(h)\right|^{2} \lesssim|n|_{\max }^{2} e^{-2\left|\beta_{2 n}\right|(h-\hat{h})}\left|\boldsymbol{u}_{n}(\hat{h})\right|^{2}
$$

By (3.15), it can be verified from $\left|\boldsymbol{\alpha}_{n}\right|^{2}>\kappa_{2}^{2}$ that

$$
\begin{aligned}
\left|M_{11}^{(n)}\right| & =\left|\alpha_{1 n}^{2}\left(\beta_{1 n}-\beta_{2 n}\right)+\beta_{2 n} \chi_{n}\right| \\
& =\left|\alpha_{1 n}^{2} \mathrm{i}\left(\left(\left|\boldsymbol{\alpha}_{n}\right|^{2}-\kappa_{1}^{2}\right)^{1 / 2}-\left(\left|\boldsymbol{\alpha}_{n}\right|^{2}-\kappa_{2}^{2}\right)^{1 / 2}\right)+\beta_{2 n} \chi_{n}\right| \\
& =\left.|| \alpha_{1 n}\right|^{2} \frac{\mathrm{i}\left(\kappa_{2}^{2}-\kappa_{1}^{2}\right)}{\left(\left|\boldsymbol{\alpha}_{n}\right|^{2}-\kappa_{1}^{2}\right)^{1 / 2}+\left(\left|\boldsymbol{\alpha}_{n}\right|^{2}-\kappa_{2}^{2}\right)^{1 / 2}}+\beta_{2 n} \chi_{n} \mid \\
& \lesssim|n|_{\max } .
\end{aligned}
$$

Following the same argument, we may show that

$$
\left|M_{i j}^{(n)}\right| \lesssim|n|_{\max }, \quad i, j=1,2,3 .
$$

Substituting (5.11), (5.12) into (3.14), we obtain

$$
\begin{aligned}
\left|\int_{\Gamma_{h}}\left(T-T_{N}\right) \boldsymbol{u} \cdot \overline{\boldsymbol{v}} \mathrm{d} s\right| & \leqslant\left|\Lambda_{1} \Lambda_{2} \sum_{|n|_{\min }>N}\left(M_{n} \boldsymbol{u}_{n}(h)\right) \cdot \overline{\boldsymbol{v}_{n}(h)}\right| \\
& \lesssim\left(\sum_{|n|_{\min }>N}|n|_{\max }\left|\boldsymbol{u}_{n}(h)\right|^{2}\right)^{1 / 2}\left(\sum_{|n|_{\min }>N}|n|_{\max }\left|\boldsymbol{v}_{n}(h)\right|^{2}\right)^{1 / 2} \\
& \lesssim\left(\sum_{|n|_{\min }>N}|n|_{\max }^{3} e^{-2\left|\beta_{2 n}\right|(h-\hat{h})}\left|\boldsymbol{u}_{n}(\hat{h})\right|^{2}\right)^{1 / 2}\|\boldsymbol{v}\|_{\boldsymbol{H}^{1 / 2}\left(\Gamma_{h}\right)}
\end{aligned}
$$




$$
\begin{aligned}
& \lesssim \max _{|n|_{\min }>N}\left(|n|_{\max } e^{-\left|\beta_{2 n}\right|(h-\hat{h})}\right)\|\boldsymbol{u}\|_{\boldsymbol{H}^{1}(\Omega)}\|\boldsymbol{v}\|_{\boldsymbol{H}^{1}(\Omega)} \\
& \lesssim \max _{|n|_{\min }>N}\left(|n|_{\max } e^{-\left|\beta_{2 n}\right|(h-\hat{h})}\right)\left\|\boldsymbol{u}^{\mathrm{inc}}\right\|_{\boldsymbol{H}^{1}(\Omega)}\|\boldsymbol{v}\|_{\boldsymbol{H}^{1}(\Omega)},
\end{aligned}
$$

which completes the proof.

Lemma 5.4. Let $\boldsymbol{v}$ be any function in $\boldsymbol{H}_{S, \mathrm{qp}}^{1}(\Omega)$, the following estimate holds:

$$
\left|a(\boldsymbol{\xi}, \boldsymbol{v})+\int_{\Gamma_{h}}\left(T-T_{N}\right) \boldsymbol{\xi} \cdot \overline{\boldsymbol{v}} \mathrm{d} s\right| \lesssim\left(\left(\sum_{K \in \mathcal{M}_{h}} \eta_{K}^{2}\right)^{1 / 2}+\max _{|n|_{\min }>N}\left(|n|_{\max } e^{-\left|\beta_{2 n}\right|(h-\hat{h})}\right)\left\|\boldsymbol{u}^{\mathrm{inc}}\right\|_{\boldsymbol{H}^{1}(\Omega)}\right)\|\boldsymbol{v}\|_{\boldsymbol{H}^{1}(\Omega)},
$$

which gives by taking $\boldsymbol{v}=\boldsymbol{\xi}$ that

$$
\begin{aligned}
\left|a(\boldsymbol{\xi}, \boldsymbol{\xi})+\int_{\Gamma_{h}}\left(T-T_{N}\right) \boldsymbol{\xi} \cdot \overline{\boldsymbol{\xi}} \mathrm{d} s\right| \lesssim & \left(\left(\sum_{K \in \mathcal{M}_{h}} \eta_{K}^{2}\right)^{1 / 2}+\left\|\boldsymbol{u}^{\mathrm{inc}}-\boldsymbol{g}^{h}\right\|_{\boldsymbol{H}^{1 / 2}(S)}\right. \\
& \left.+\max _{|n|_{\min }>N}\left(|n|_{\max } e^{-\left|\beta_{2 n}\right|(h-\hat{h})}\right)\left\|\boldsymbol{u}^{\mathrm{inc}}\right\|_{\boldsymbol{H}^{1}(\Omega)}\right)\|\boldsymbol{\xi}\|_{\boldsymbol{H}^{1}(\Omega)} .
\end{aligned}
$$

Since the proof of Lemma 5.4 is essentially the same as that for Lemma 5.4 of [32], we omit it for brevity. The following two lemmas are to estimate the last term in (5.3).

Let $\hat{M}_{n}=-\frac{1}{2}\left(M_{n}+M_{n}^{*}\right)$. Note that $\hat{M}_{n}=\Re\left(-\mathrm{i} W_{n}\right)$ which is defined in (3.13) of [20]. By Lemma 2 of [20], we have the following lemma.

Lemma 5.5. $\hat{M}_{n}$ is positive definite for $\left|\boldsymbol{\alpha}_{n}\right|>\kappa_{2}$.

Lemma 5.6. Let $\Omega^{\prime}=\left\{\boldsymbol{x} \in \mathbb{R}^{3}:\left(x_{1}, x_{2}\right) \in\left(0, \Lambda_{1}\right) \times\left(0, \Lambda_{2}\right), \hat{h}<x_{3}<h\right\}$. Then for any $\delta>0$, there exists a positive constant $C(\delta)$ independent of $N$ such that

$$
\Re \int_{\Gamma_{h}} T_{N} \boldsymbol{\xi} \cdot \overline{\boldsymbol{\xi}} \mathrm{d} s \leqslant C(\delta)\|\boldsymbol{\xi}\|_{\boldsymbol{L}^{2}\left(\Omega^{\prime}\right)}^{2}+\delta\|\boldsymbol{\xi}\|_{\boldsymbol{H}^{1}\left(\Omega^{\prime}\right)}^{2} .
$$

Proof. It follows from the definition of the DtN operator (3.14) that we have

$$
\begin{aligned}
\Re \int_{\Gamma_{h}} T_{N} \boldsymbol{\xi} \cdot \overline{\boldsymbol{\xi}} \mathrm{d} s & =\Lambda_{1} \Lambda_{2} \Re \sum_{\left|n_{1}\right|,\left|n_{2}\right| \leqslant N}\left(M_{n} \boldsymbol{\xi}_{n}\right) \cdot \overline{\boldsymbol{\xi}_{n}} \\
& =-\Lambda_{1} \Lambda_{2} \sum_{\left|n_{1}\right|,\left|n_{2}\right| \leqslant N}\left(\hat{M}_{n} \boldsymbol{\xi}_{n}\right) \cdot \overline{\boldsymbol{\xi}_{n}}
\end{aligned}
$$

By Lemma 5.5, $\hat{M}_{n}$ is positive definite for sufficiently large $|n|_{\max }$. Hence for fixed $\omega, \lambda, \mu$, there exists a positive integer $N^{*}$ such that

$$
\Re \int_{\Gamma_{h}} T_{N} \boldsymbol{\xi} \cdot \overline{\boldsymbol{\xi}} \mathrm{d} s \leqslant-\Lambda_{1} \Lambda_{2} \sum_{|n|_{\max } \leqslant \min \left(N, N^{*}\right)}\left(\hat{M}_{n} \boldsymbol{\xi}_{n}\right) \cdot \overline{\boldsymbol{\xi}_{n}} \quad \forall|n|_{\max }>N^{*}
$$

On the other hand, there exists a constant $C$ depending only on $\omega, \mu, \lambda$ such that

$$
\left|\left(\hat{M}_{n} \boldsymbol{\xi}_{n}\right) \cdot \overline{\boldsymbol{\xi}_{n}}\right| \leqslant C\left|\boldsymbol{\xi}_{n}\right|^{2} \quad \forall|n|_{\max } \leqslant \min \left(N^{*}, N\right) .
$$


For any $\delta>0$, it follows from Young's inequality that

$$
\begin{aligned}
(h-\hat{h})|\phi(h)|^{2} & =\int_{\hat{h}}^{h}\left|\phi\left(x_{3}\right)\right|^{2} \mathrm{~d} x_{3}+\int_{\hat{h}}^{h} \int_{x_{3}}^{h}\left(|\phi(s)|^{2}\right)^{\prime} \mathrm{d} s \mathrm{~d} x_{3} \\
& \leqslant \int_{\hat{h}}^{h}\left|\phi\left(x_{3}\right)\right|^{2} \mathrm{~d} x_{3}+\left(\frac{h-\hat{h}}{\delta}\right) \int_{\hat{h}}^{h}\left|\phi\left(x_{3}\right)\right|^{2} \mathrm{~d} x_{3}+\delta(h-\hat{h}) \int_{\hat{h}}^{h}\left|\phi^{\prime}\left(x_{3}\right)\right|^{2} \mathrm{~d} x_{3},
\end{aligned}
$$

which gives that

$$
|\phi(h)|^{2} \leqslant\left[\delta^{-1}+(h-\hat{h})^{-1}\right] \int_{\hat{h}}^{h}\left|\phi\left(x_{3}\right)\right|^{2} \mathrm{~d} x_{3}+\delta \int_{\hat{h}}^{h}\left|\phi^{\prime}\left(x_{3}\right)\right|^{2} \mathrm{~d} x_{3} .
$$

Let $\phi(\boldsymbol{x})=\sum_{n \in \mathbb{Z}^{2}} \phi_{n}\left(x_{3}\right) e^{\mathrm{i} \boldsymbol{\alpha}_{n} \cdot \boldsymbol{r}}$. It is easy to get

$$
\begin{aligned}
\|\nabla \phi\|_{L^{2}\left(\Omega^{\prime}\right)}^{2} & =\Lambda_{1} \Lambda_{2} \sum_{n \in \mathbb{Z}^{2}} \int_{\hat{h}}^{h}\left(\left|\phi_{n}^{\prime}\left(x_{3}\right)\right|^{2}+\left|\boldsymbol{\alpha}_{n}\right|^{2}\left|\phi_{n}\left(x_{3}\right)\right|^{2}\right) \mathrm{d} x_{3}, \\
\|\phi\|_{L^{2}\left(\Omega^{\prime}\right)}^{2} & =\Lambda_{1} \Lambda_{2} \sum_{n \in \mathbb{Z}^{2}} \int_{\hat{h}}^{h}\left|\phi_{n}\left(x_{3}\right)\right|^{2} \mathrm{~d} x_{3} .
\end{aligned}
$$

Hence, we have for any $\phi \in \boldsymbol{H}^{1}\left(\Omega^{\prime}\right)$ that

$$
\begin{aligned}
\|\phi\|_{L^{2}\left(\Gamma_{h}\right)}^{2} & =\Lambda_{1} \Lambda_{2} \sum_{n \in \mathbb{Z}^{2}}\left|\phi_{n}(h)\right|^{2} \\
& \leqslant \Lambda_{1} \Lambda_{2}\left[\delta^{-1}+(h-\hat{h})^{-1}\right] \sum_{n \in \mathbb{Z}^{2}} \int_{\hat{h}}^{h}\left|\phi_{n}\left(x_{3}\right)\right|^{2} \mathrm{~d} x_{3}+\Lambda_{1} \Lambda_{2} \delta \sum_{n \in \mathbb{Z}^{2}} \int_{\hat{h}}^{h}\left|\phi_{n}^{\prime}\left(x_{3}\right)\right|^{2} \mathrm{~d} x_{3} \\
& \leqslant \Lambda_{1} \Lambda_{2}\left[\frac{1}{\delta}+\left(h_{1}-h_{2}\right)^{-1}\right] \sum_{n \in \mathbb{Z}^{2}} \int_{\hat{h}}^{h}\left|\phi_{n}\left(x_{3}\right)\right|^{2} \mathrm{~d} x_{3}+\Lambda_{1} \Lambda_{2} \delta \sum_{n \in \mathbb{Z}^{2}} \int_{\hat{h}}^{h}\left[\left|\phi_{n}^{\prime}\left(x_{3}\right)\right|^{2}+\left|\boldsymbol{\alpha}_{n}\right|^{2}\left|\phi_{n}\left(x_{3}\right)\right|^{2}\right] \mathrm{d} x_{3} \\
& \leqslant\left[\delta^{-1}+(h-\hat{h})^{-1}\right]\|\phi\|_{L^{2}\left(\Omega^{\prime}\right)}^{2}+\delta\|\nabla \phi\|_{L^{2}\left(\Omega^{\prime}\right)}^{2} \\
& \leqslant C(\delta)\|\phi\|_{L^{2}\left(\Omega^{\prime}\right)}^{2}+\delta\|\nabla \phi\|_{L^{2}\left(\Omega^{\prime}\right)}^{2} .
\end{aligned}
$$

Combining the above estimates, we obtain

$$
\Re \int_{\Gamma_{h}} T_{N} \boldsymbol{\xi} \cdot \overline{\boldsymbol{\xi}} \mathrm{d} s \leqslant C\|\boldsymbol{\xi}\|_{\boldsymbol{L}^{2}\left(\Gamma_{h}\right)}^{2} \leqslant C(\delta)\|\boldsymbol{\xi}\|_{\boldsymbol{L}^{2}\left(\Omega^{\prime}\right)}^{2}+\delta\|\boldsymbol{\xi}\|_{\boldsymbol{H}^{1}\left(\Omega^{\prime}\right)}^{2},
$$

which completes the proof.

To estimate the third term of (5.3), we introduce the dual problem

$$
a(\boldsymbol{v}, \boldsymbol{p})=\int_{\Omega} \boldsymbol{v} \cdot \overline{\boldsymbol{\xi}} \mathrm{d} \boldsymbol{x} \quad \forall \boldsymbol{v} \in H_{S, \mathrm{qp}}^{1}(\Omega) .
$$

It is easy to check that $\boldsymbol{p}$ is the weak solution of the boundary value problem

$$
\begin{cases}\mu \Delta \boldsymbol{p}+(\lambda+\mu) \nabla \nabla \cdot \boldsymbol{p}+\omega^{2} \boldsymbol{p}=-\boldsymbol{\xi} & \text { in } \Omega \\ \boldsymbol{p}=0 & \text { on } S \\ D \boldsymbol{p}=T^{*} \boldsymbol{p} & \text { on } \Gamma_{h}\end{cases}
$$


where $T^{*}$ is the adjoint operator to $T$ under the scalar product in $L^{2}\left(\Gamma_{h}\right)$. Taking $\boldsymbol{v}=\boldsymbol{\xi}$ in (5.13), we have

$$
\|\boldsymbol{\xi}\|_{\boldsymbol{L}^{2}(\Omega)}^{2}=a(\boldsymbol{\xi}, \boldsymbol{p})-\int_{\Gamma_{h}}\left(T-T_{N}\right) \boldsymbol{\xi} \cdot \overline{\boldsymbol{p}} \mathrm{d} s+\int_{\Gamma_{h}}\left(T-T_{N}\right) \boldsymbol{\xi} \cdot \overline{\boldsymbol{p}} \mathrm{d} s
$$

It is clear that the evaluation of $\boldsymbol{p}$ is essential to the error estimate. Lemmas $5.7-5.9$ give the asymptotic analysis of $\boldsymbol{p}$. First, we introduce the Helmholtz decomposition of $\boldsymbol{\xi}$ in $\Omega^{\prime}$ :

$$
\boldsymbol{\xi}=\nabla \zeta+\nabla \times \boldsymbol{Z}, \quad \nabla \cdot \boldsymbol{Z}=0
$$

where $\boldsymbol{Z}=\left(Z_{1}, Z_{2}, Z_{3}\right)^{\top}$ and

$$
\zeta(\boldsymbol{x})=\sum_{\boldsymbol{n} \in \mathbb{Z}^{2}} \zeta_{n}\left(x_{3}\right) e^{\mathrm{i} \boldsymbol{\alpha}_{n} \cdot \boldsymbol{r}}, \quad Z_{j}(\boldsymbol{x})=\sum_{\boldsymbol{n} \in \mathbb{Z}^{2}} Z_{j n}\left(x_{3}\right) e^{\mathrm{i} \boldsymbol{\alpha}_{n} \cdot \boldsymbol{r}} .
$$

Substituting the above Fourier series expansions into (5.16) gives

$$
\left[\begin{array}{c}
Z_{1 n}^{\prime}\left(x_{3}\right) \\
Z_{2 n}^{\prime}\left(x_{3}\right) \\
Z_{3 n}^{\prime}\left(x_{3}\right) \\
\zeta_{n}^{\prime}\left(x_{3}\right)
\end{array}\right]=\left[\begin{array}{cccc}
0 & 0 & \mathrm{i} \alpha_{1 n} & -\mathrm{i} \alpha_{2 n} \\
0 & 0 & \mathrm{i} \alpha_{2 n} & \mathrm{i} \alpha_{1 n} \\
-\mathrm{i} \alpha_{1 n} & -\mathrm{i} \alpha_{2 n} & 0 & 0 \\
\mathrm{i} \alpha_{2 n} & -\mathrm{i} \alpha_{1 n} & 0 & 0
\end{array}\right]\left[\begin{array}{c}
Z_{1 n}\left(x_{3}\right) \\
Z_{2 n}\left(x_{3}\right) \\
Z_{3 n}\left(x_{3}\right) \\
\zeta_{n}\left(x_{3}\right)
\end{array}\right]+\left[\begin{array}{c}
\xi_{2 n}\left(x_{3}\right) \\
-\xi_{1 n}\left(x_{3}\right) \\
0 \\
\xi_{3 n}\left(x_{3}\right)
\end{array}\right] .
$$

In addition, the homogeneous Dirichlet boundary condition is imposed for the Fourier coefficients at $x_{3}=h$ :

$$
Z_{1 n}(h)=Z_{2 n}(h)=Z_{3 n}(h)=\zeta_{n}(h)=0 .
$$

Lemma 5.7. The solutions of the problem (5.17) and (5.18) in $[\hat{h}, h]$ satisfy the following estimates:

$$
\begin{aligned}
\left|\zeta_{n}\left(x_{3}\right)\right| & \lesssim\left\|\boldsymbol{\xi}_{n}\right\|_{\boldsymbol{L}^{\infty}([\hat{h}, h])} \frac{1}{\left|\boldsymbol{\alpha}_{n}\right|} e^{\left|\boldsymbol{\alpha}_{n}\right|\left(h-x_{3}\right)}, \\
\left|Z_{j n}\left(x_{3}\right)\right| & \lesssim\left\|\boldsymbol{\xi}_{n}\right\|_{\boldsymbol{L}^{\infty}([\hat{h}, h])} \frac{1}{\left|\boldsymbol{\alpha}_{n}\right|} e^{\left|\boldsymbol{\alpha}_{n}\right|\left(h-x_{3}\right)}, \quad j=1,2,3 .
\end{aligned}
$$

Proof. A straight forward calculation shows that the solution of problem (5.17) and (5.18) is

$$
\begin{aligned}
Z_{1 n}\left(x_{3}\right)= & -\frac{1}{2} e^{\left|\boldsymbol{\alpha}_{n}\right| x_{3}} \int_{x_{3}}^{h} e^{-\left|\boldsymbol{\alpha}_{n}\right| t} \xi_{2 n}(t) \mathrm{d} t-\frac{1}{2} e^{-\left|\boldsymbol{\alpha}_{n}\right| x_{3}} \int_{x_{3}}^{h} e^{\left|\boldsymbol{\alpha}_{n}\right| t} \xi_{2 n}(t) \mathrm{d} t \\
& +\frac{\mathrm{i}}{2} \frac{\alpha_{2 n}}{\left|\boldsymbol{\alpha}_{n}\right|} e^{\left|\boldsymbol{\alpha}_{n}\right| x_{3}} \int_{x_{3}}^{h} e^{-\left|\boldsymbol{\alpha}_{n}\right| t} \xi_{3 n}(t) \mathrm{d} t-\frac{\mathrm{i}}{2} \frac{\alpha_{2 n}}{\left|\boldsymbol{\alpha}_{n}\right|} e^{-\left|\boldsymbol{\alpha}_{n}\right| x_{3}} \int_{x_{3}}^{h} e^{\left|\boldsymbol{\alpha}_{n}\right| t} \xi_{3 n}(t) \mathrm{d} t, \\
Z_{2 n}\left(x_{3}\right)= & \frac{1}{2} e^{\left|\boldsymbol{\alpha}_{n}\right| x_{3}} \int_{x_{3}}^{h} e^{-\left|\boldsymbol{\alpha}_{n}\right| t} \xi_{1 n}(t) \mathrm{d} t+\frac{1}{2} e^{-\left|\boldsymbol{\alpha}_{n}\right| x_{3}} \int_{x_{3}}^{h} e^{\left|\boldsymbol{\alpha}_{n}\right| t} \xi_{1 n}(t) \mathrm{d} t \\
& -\frac{\mathrm{i}}{2} \frac{\alpha_{1 n}}{\left|\boldsymbol{\alpha}_{n}\right|} e^{\left|\boldsymbol{\alpha}_{n}\right| x_{3}} \int_{x_{3}}^{h} e^{-\left|\boldsymbol{\alpha}_{n}\right| t} \xi_{3 n}(t) \mathrm{d} t+\frac{\mathrm{i}}{2} \frac{\alpha_{1 n}}{\left|\boldsymbol{\alpha}_{n}\right|} e^{-\left|\boldsymbol{\alpha}_{n}\right| x_{3}} \int_{x_{3}}^{h} e^{\left|\boldsymbol{\alpha}_{n}\right| t} \xi_{3 n}(t) \mathrm{d} t, \\
Z_{3 n}\left(x_{3}\right)= & \frac{\mathrm{i}}{2} \frac{\alpha_{1 n}}{\left|\boldsymbol{\alpha}_{n}\right|} e^{\left|\boldsymbol{\alpha}_{n}\right| x_{3}} \int_{x_{3}}^{h} e^{-\left|\boldsymbol{\alpha}_{n}\right| t} \xi_{2 n}(t) \mathrm{d} t-\frac{\mathrm{i}}{2} \frac{\alpha_{1 n}}{\left|\boldsymbol{\alpha}_{n}\right|} e^{-\left|\boldsymbol{\alpha}_{n}\right| x_{3}} \int_{x_{3}}^{h} e^{\left|\boldsymbol{\alpha}_{n}\right| t} \xi_{2 n}(t) \mathrm{d} t \\
& -\frac{\mathrm{i}}{2} \frac{\alpha_{2 n}}{\left|\boldsymbol{\alpha}_{n}\right|} e^{\left|\boldsymbol{\alpha}_{n}\right| x_{3}} \int_{x_{3}}^{h} e^{-\left|\boldsymbol{\alpha}_{n}\right| t} \xi_{1 n}(t) \mathrm{d} t+\frac{\mathrm{i}}{2} \frac{\alpha_{2 n}}{\left|\boldsymbol{\alpha}_{n}\right|} e^{-\left|\boldsymbol{\alpha}_{n}\right| x_{3}} \int_{x_{3}}^{h} e^{\left|\boldsymbol{\alpha}_{n}\right| t} \xi_{1 n}(t) \mathrm{d} t
\end{aligned}
$$


and

$$
\begin{aligned}
\zeta_{n}\left(x_{3}\right)= & -\frac{1}{2} e^{\left|\boldsymbol{\alpha}_{n}\right| x_{3}} \int_{x_{3}}^{h} e^{-\left|\boldsymbol{\alpha}_{n}\right| t} \xi_{3 n}(t) \mathrm{d} t-\frac{1}{2} e^{-\left|\boldsymbol{\alpha}_{n}\right| x_{3}} \int_{x_{3}}^{h} e^{\left|\boldsymbol{\alpha}_{n}\right| t} \xi_{3 n}(t) \mathrm{d} t \\
& -\frac{\mathrm{i}}{2} \frac{\alpha_{2 n}}{\left|\boldsymbol{\alpha}_{n}\right|} e^{\left|\boldsymbol{\alpha}_{n}\right| x_{3}} \int_{x_{3}}^{h} e^{-\left|\boldsymbol{\alpha}_{n}\right| t} \xi_{2 n}(t) \mathrm{d} t+\frac{\mathrm{i}}{2} \frac{\alpha_{2 n}}{\left|\boldsymbol{\alpha}_{n}\right|} e^{-\left|\boldsymbol{\alpha}_{n}\right| x_{3}} \int_{x_{3}}^{h} e^{\left|\boldsymbol{\alpha}_{n}\right| t} \xi_{2 n}(t) \mathrm{d} t \\
& -\frac{\mathrm{i}}{2} \frac{\alpha_{1 n}}{\left|\boldsymbol{\alpha}_{n}\right|} e^{\left|\boldsymbol{\alpha}_{n}\right| x_{3}} \int_{x_{3}}^{h} e^{-\left|\boldsymbol{\alpha}_{n}\right| t} \xi_{1 n}(t) \mathrm{d} t+\frac{\mathrm{i}}{2} \frac{\alpha_{1 n}}{\left|\boldsymbol{\alpha}_{n}\right|} e^{-\left|\boldsymbol{\alpha}_{n}\right| x_{3}} \int_{x_{3}}^{h} e^{\left|\boldsymbol{\alpha}_{n}\right| t} \xi_{1 n}(t) \mathrm{d} t .
\end{aligned}
$$

It is easy to check from (5.19)-(5.22) that

$$
\begin{aligned}
\left|\zeta_{n}\left(x_{3}\right)\right| & \lesssim\left\|\boldsymbol{\xi}_{n}\right\|_{\boldsymbol{L}^{\infty}([\hat{h}, h])} \frac{1}{\left|\boldsymbol{\alpha}_{n}\right|} e^{\left|\boldsymbol{\alpha}_{n}\right|\left(h-x_{3}\right)}, \\
\left|Z_{j n}\left(x_{3}\right)\right| & \lesssim\left\|\boldsymbol{\xi}_{n}\right\|_{\boldsymbol{L}^{\infty}([\hat{h}, h]) \frac{1}{\left|\boldsymbol{\alpha}_{n}\right|} e^{\left|\boldsymbol{\alpha}_{n}\right|\left(h-x_{3}\right)}, \quad j=1,2,3,}
\end{aligned}
$$

which complete the proof.

Consider the following boundary value problem for $\boldsymbol{p}$ in $\Omega^{\prime}$ :

$$
\begin{cases}\mu \Delta \boldsymbol{p}+(\lambda+\mu) \nabla \nabla \cdot \boldsymbol{p}+\omega^{2} \boldsymbol{p}=-\boldsymbol{\xi} & \text { in } \Omega^{\prime}, \\ \boldsymbol{p}=\boldsymbol{p} & \text { on } \Gamma_{\hat{h}}, \\ D \boldsymbol{p}=T^{*} \boldsymbol{p} & \text { on } \Gamma_{h} .\end{cases}
$$

Lemma 5.8. Let $\boldsymbol{q}=\left(q_{1}, q_{2}, q_{3}\right)^{\top}$ and $g$ have the Fourier series expansions

$$
q_{j}(\boldsymbol{x})=\sum_{n \in \mathbb{Z}^{2}} q_{j n}\left(x_{3}\right) e^{\mathrm{i} \boldsymbol{\alpha}_{n} \cdot \boldsymbol{r}}, \quad g(\boldsymbol{x})=\sum_{n \in \mathbb{Z}^{2}} g_{n}\left(x_{3}\right) e^{\mathrm{i} \boldsymbol{\alpha}_{n} \cdot \boldsymbol{r}}, \quad \boldsymbol{x} \in \Omega^{\prime}
$$

and satisfy

$$
\begin{cases}(\lambda+2 \mu)\left(\Delta g+\kappa_{1}^{2} g\right)=-\zeta & \text { in } \Omega^{\prime}, \\ \mu\left(\nabla \times(\nabla \times \boldsymbol{q})-\kappa_{2}^{2} \boldsymbol{q}\right)=\boldsymbol{Z}, \quad \nabla \cdot \boldsymbol{q}=0 & \text { in } \Omega^{\prime}, \\ \boldsymbol{q}=\boldsymbol{q}, \quad g=g & \text { on } \Gamma_{\hat{h}} .\end{cases}
$$

Moreover, the Fourier coefficients are assumed to satisfy the following boundary conditions on $\Gamma_{h}$ :

$$
g_{n}^{\prime}(h)=-\mathrm{i} \overline{\beta_{1 n}} g_{n}(h)
$$

and

$$
\begin{aligned}
& q_{1 n}^{\prime}(h)=-\mathrm{i} \overline{\beta_{2 n}} q_{1 n}(h), \quad q_{2 n}^{\prime}(h)=-\mathrm{i} \overline{\beta_{2 n}} q_{2 n}(h), \\
& q_{3 n}^{\prime}(h)=\mathrm{i} \alpha_{1 n} q_{1 n}(h)+\mathrm{i} \alpha_{2 n} q_{2 n}(h) .
\end{aligned}
$$

Then $\boldsymbol{p}$ has Helmholtz decomposition $\boldsymbol{p}=\nabla g+\nabla \times \boldsymbol{q}$ and satisfies boundary value problem (5.23).

Proof. Substituting $\boldsymbol{p}=\nabla g+\nabla \times \boldsymbol{q}$ into the elastic wave equation, we obtain

$$
\begin{aligned}
\mu \Delta & (\nabla g+\nabla \times \boldsymbol{q})+(\lambda+\mu) \nabla \nabla \cdot(\nabla g+\nabla \times \boldsymbol{q})+\omega^{2}(\nabla g+\nabla \times \boldsymbol{q}) \\
& =\nabla\left(\mu \Delta g+(\lambda+\mu) \Delta g+\omega^{2} g\right)+\nabla \times\left(\mu \Delta \boldsymbol{q}+\omega^{2} \boldsymbol{q}\right) \\
& =(\lambda+2 \mu) \nabla\left(\Delta g+\kappa_{1}^{2} g\right)+\mu \nabla \times\left(-\nabla \times(\nabla \times \boldsymbol{q})+\kappa_{2}^{2} \boldsymbol{q}\right) \\
& =-\nabla \zeta-\nabla \times \boldsymbol{Z}=-\boldsymbol{\xi} .
\end{aligned}
$$


Since $g(\boldsymbol{x})=\sum_{n \in \mathbb{Z}^{2}} g_{n}\left(x_{3}\right) e^{\mathrm{i} \boldsymbol{\alpha}_{n} \cdot \boldsymbol{r}}$, we get from taking the second order partial derivatives of $g$ that

$$
\begin{aligned}
& \partial_{x_{1}}^{2} g(\boldsymbol{x})=-\sum_{n \in \mathbb{Z}} \alpha_{1 n}^{2} g_{n}\left(x_{3}\right) e^{\mathrm{i} \boldsymbol{\alpha}_{n} \cdot \boldsymbol{r}}, \\
& \partial_{x_{2}}^{2} g(\boldsymbol{x})=-\sum_{n \in \mathbb{Z}} \alpha_{2 n}^{2} g_{n}\left(x_{3}\right) e^{\mathrm{i} \boldsymbol{\alpha}_{n} \cdot \boldsymbol{r}}, \\
& \partial_{x_{3}}^{2} g(\boldsymbol{x})=\sum_{n \in \mathbb{Z}} g_{n}^{\prime \prime}\left(x_{3}\right) e^{\mathrm{i} \boldsymbol{\alpha}_{n} \cdot \boldsymbol{r}} .
\end{aligned}
$$

Substituting the above three expansions into $(\lambda+2 \mu)\left(\Delta g+\kappa_{1}^{2} g\right)=-\zeta$ yields

$$
g_{n}^{\prime \prime}\left(x_{3}\right)-\left|\boldsymbol{\alpha}_{n}\right|^{2} g_{n}\left(x_{3}\right)+\kappa_{1}^{2} g_{n}\left(x_{3}\right)=-\frac{1}{\lambda+2 \mu} \zeta_{n}\left(x_{3}\right) .
$$

Similarly, we may verify that $q_{j n}$ satisfies the second ordinary differential equation

$$
q_{j n}^{\prime \prime}\left(x_{3}\right)-\left|\boldsymbol{\alpha}_{n}\right|^{2} q_{j n}\left(x_{3}\right)+\kappa_{2}^{2} q_{j n}\left(x_{3}\right)=-\frac{1}{\mu} Z_{j n}\left(x_{3}\right) .
$$

Letting $\boldsymbol{p}=\sum_{n \in \mathbb{Z}^{2}} \boldsymbol{p}_{n}\left(x_{3}\right) e^{\mathrm{i} \boldsymbol{\alpha}_{n} \cdot \boldsymbol{r}}$ and plugging it into $\boldsymbol{p}=\nabla g+\nabla \times \boldsymbol{q}$, we get

$$
\left[\begin{array}{l}
p_{1 n}\left(x_{3}\right) \\
p_{2 n}\left(x_{3}\right) \\
p_{3 n}\left(x_{3}\right)
\end{array}\right]=\left[\begin{array}{c}
\mathrm{i} \alpha_{1 n} g^{(\boldsymbol{n})}\left(x_{3}\right)+\mathrm{i} \alpha_{2 n} q_{3 n}\left(x_{3}\right)-q_{2 n}^{\prime}\left(x_{3}\right) \\
\mathrm{i} \alpha_{2 n} g_{n}\left(x_{3}\right)-\mathrm{i} \alpha_{1 n} q_{3 n}\left(x_{3}\right)+q_{1 n}^{\prime}\left(x_{3}\right) \\
\mathrm{i} \alpha_{1 n} q_{2 n}\left(x_{3}\right)-\mathrm{i} \alpha_{2 n} q_{1 n}\left(x_{3}\right)+g_{n}^{\prime}\left(x_{3}\right)
\end{array}\right] .
$$

Substituting the above expressions into the boundary operator (3.13) gives

$$
\begin{aligned}
\mu \partial_{x_{3}} \boldsymbol{p}+(\lambda+\mu)(0,0,1)^{\top} \nabla \cdot \boldsymbol{p} \\
=\sum_{n \in \mathbb{Z}^{2}} e^{\mathrm{i} \boldsymbol{\alpha}_{n} \cdot \boldsymbol{r}}\left[\begin{array}{c}
\mu p_{1 n}^{\prime} \\
\mu p_{2 n}^{\prime} \\
\mu p_{3 n}^{\prime}+(\lambda+\mu) \mathrm{i} \alpha_{1 n} p_{1 n}+(\lambda+\mu) \mathrm{i} \alpha_{2 n} p_{2 n}+(\lambda+\mu) p_{3 n}^{\prime}
\end{array}\right] \\
=\sum_{n \in \mathbb{Z}^{2}} e^{\mathrm{i} \boldsymbol{\alpha}_{n} \cdot \boldsymbol{r}}\left[\begin{array}{c}
\left.\mathrm{i} \alpha_{1 n} g_{n}^{\prime}+\mathrm{i} \alpha_{2 n} q_{3 n}^{\prime}-q_{2 n}^{\prime \prime}\right) \\
\mu\left(\mathrm{i} \alpha_{2 n} g_{n}^{\prime}-\mathrm{i} \alpha_{1 n} q_{3 n}^{\prime}+q_{1 n}^{\prime \prime}\right) \\
(\lambda+2 \mu)\left(\mathrm{i} \alpha_{1 n} q_{2 n}^{\prime}-\mathrm{i} \alpha_{2 n} q_{1 n}^{\prime}+g_{n}^{\prime \prime}\right)+(\lambda+\mu)\left(\mathrm{i} \alpha_{1 n} p_{1 n}+\mathrm{i} \alpha_{2 n} p_{2 n}\right)
\end{array}\right] \\
\quad \begin{array}{c}
\mathrm{i} \mu \alpha_{1 n} g_{n}^{\prime}+\mathrm{i} \mu \alpha_{2 n} q_{3 n}^{\prime}+Z_{2 n}+\mu\left(\kappa_{2}^{2}-\left|\boldsymbol{\alpha}_{n}\right|^{2}\right) q_{2 n} \\
\mathrm{i} \mu \alpha_{2 n} g_{n}^{\prime}-\mathrm{i} \mu \alpha_{1 n} q_{3 n}^{\prime}-Z_{1 n}-\mu\left(\kappa_{2}^{2}-\left|\boldsymbol{\alpha}_{n}\right|^{2}\right) q_{1 n}
\end{array} \\
=\sum_{n \in \mathbb{Z}^{2}} e^{\mathrm{i} \boldsymbol{\alpha}_{n} \cdot \boldsymbol{r}}\left[\begin{array}{c}
\left.\left.\boldsymbol{\alpha}_{n}\right|^{2}\right) g_{n}+\mathrm{i} \mu \alpha_{1 n} q_{2 n}^{\prime}-\mathrm{i} \mu \alpha_{2 n} q_{1 n}^{\prime}-(\lambda+\mu)\left|\boldsymbol{\alpha}_{n}\right|^{2} g_{n}
\end{array}\right] .
\end{aligned}
$$

Substituting (5.18) and (5.25), (5.26) into the above equation and evaluating it at $x_{3}=h$, we get

$$
\begin{aligned}
D \boldsymbol{p} & =\mu \partial_{x_{3}} \boldsymbol{p}+(\lambda+\mu)(0,0,1)^{\top} \nabla \cdot \boldsymbol{p} \\
& =\sum_{n \in \mathbb{Z}^{2}} e^{\mathrm{i} \boldsymbol{\alpha}_{n} \cdot \boldsymbol{r}}\left[\begin{array}{ccc}
-\mu \alpha_{1 n} \alpha_{2 n} & \mu\left(\beta_{2 n}^{2}-\alpha_{2 n}^{2}\right) & \mu \alpha_{1 n} \overline{\beta_{1 n}} \\
-\mu\left(\beta_{2 n}^{2}-\alpha_{2 n}^{2}\right) & \mu \alpha_{1 n} \alpha_{2 n} & \mu \alpha_{2 n} \overline{\beta_{1 n}} \\
-\mu \alpha_{2 n} \overline{\beta_{2 n}} & \mu \alpha_{1 n} \overline{\beta_{2 n}} & -\mu \kappa_{2}^{2}+\mu\left|\boldsymbol{\alpha}_{n}\right|^{2}
\end{array}\right]\left[\begin{array}{c}
q_{1 n}(h) \\
q_{2 n}(h) \\
g_{n}(h)
\end{array}\right] .
\end{aligned}
$$

On the other hand, substituting (5.29) into (3.15) gives

$$
T^{*} \boldsymbol{p}=\sum_{n \in \mathbb{Z}^{2}} M_{n}^{*} \boldsymbol{p}_{n}(h) e^{\mathrm{i} \boldsymbol{\alpha}_{n} \cdot \boldsymbol{r}}
$$




$$
=\sum_{n \in \mathbb{Z}^{2}} M_{n}^{*}\left[\begin{array}{cccc}
0 & \mathrm{i} \overline{\beta_{2 n}} & \mathrm{i} \alpha_{2 n} & \mathrm{i} \alpha_{1 n} \\
-\mathrm{i} \overline{\beta_{2 n}} & 0 & -\mathrm{i} \alpha_{1 n} & \mathrm{i} \alpha_{2 n} \\
-\mathrm{i} \alpha_{2 n} & \mathrm{i} \alpha_{1 n} & 0 & -\mathrm{i} \overline{\beta_{1 n}}
\end{array}\right]\left[\begin{array}{c}
q_{1 n}(h) \\
q_{2 n}(h) \\
q_{3 n}(h) \\
g_{n}(h)
\end{array}\right]=\sum_{n \in \mathbb{Z}^{2}} K_{n}\left[\begin{array}{c}
q_{1 n}(h) \\
q_{2 n}(h) \\
q_{3 n}(h) \\
g_{n}(h)
\end{array}\right]
$$

where

$$
K_{n}=-\mathrm{i} \mu\left[\begin{array}{cccc}
0 & \mathrm{i} \overline{\beta_{2 n}^{2}} & \mathrm{i} \overline{\beta_{2 n}} \alpha_{2 n} & \mathrm{i} \overline{\beta_{1 n}} \alpha_{1 n} \\
-\mathrm{i} \overline{\beta_{2 n}^{2}} & 0 & -\mathrm{i} \overline{\beta_{2 n}} \alpha_{1 n} & \mathrm{i} \overline{\beta_{1 n}} \alpha_{2 n} \\
-\mathrm{i} \overline{\beta_{2 n}} \alpha_{2 n} & \mathrm{i} \overline{\beta_{2 n}} \alpha_{1 n} & 0 & -\mathrm{i} \overline{\beta_{2 n}^{2}}
\end{array}\right] .
$$

It follows from $\nabla \cdot \boldsymbol{q}=0$ that

$$
q_{3 n}^{\prime}\left(x_{3}\right)=\mathrm{i} \alpha_{1 n} q_{1 n}\left(x_{3}\right)+\mathrm{i} \alpha_{2 n} q_{2 n}\left(x_{3}\right) .
$$

Taking the derivative of the above equation and combining the result with (5.28), we get

$$
\mathrm{i} \alpha_{1 n} q_{1 n}^{\prime}\left(x_{3}\right)+\mathrm{i} \alpha_{2 n} q_{2 n}^{\prime}\left(x_{3}\right)=-\frac{1}{\mu} Z_{3 n}\left(x_{3}\right)-\left(\kappa_{2}^{2}-\left|\boldsymbol{\alpha}_{n}\right|^{2}\right) q_{3 n}\left(x_{3}\right) .
$$

Evaluating $q_{3 n}\left(x_{3}\right)$ at $x_{3}=h$, we have from (5.25) and (5.26) that

$$
-\left(\kappa_{2}^{2}-\left|\boldsymbol{\alpha}_{n}\right|^{2}\right) q_{3 n}(h)=\mathrm{i} \alpha_{1 n}\left(-\mathrm{i} \overline{\beta_{2 n}}\right) q_{1 n}(h)+\mathrm{i} \alpha_{2 n}\left(-\mathrm{i} \overline{\beta_{2 n}}\right) q_{2 n}(h),
$$

which gives

$$
q_{3 n}(h)=-\frac{\alpha_{1 n} \overline{\beta_{2 n}}}{\beta_{2 n}^{2}} q_{1 n}(h)-\frac{\alpha_{2 n} \overline{\beta_{2 n}}}{\beta_{2 n}^{2}} q_{2 n}(h) .
$$

Substituting (5.32) into (5.30), we obtain

$$
D \boldsymbol{p}=T^{*} \boldsymbol{p} \quad \text { on } \Gamma_{h},
$$

which completes the proof.

Consider the general two-point boundary value problem for the second order ordinary differential equation

$$
\left\{\begin{array}{l}
u^{\prime \prime}(y)-|\beta|^{2} u(y)=-c \xi, \quad y \in(\hat{h}, h) \\
u(\hat{h})=u(\hat{h}), \quad u^{\prime}(h)=-|\beta| u(h)
\end{array}\right.
$$

which has a unique solution given by

$$
u(y)=\frac{1}{2|\beta|}\left[-c \int_{h}^{y} e^{|\beta|(y-s)} \xi(s) \mathrm{d} s+c \int_{\hat{h}}^{y} e^{|\beta|(s-y)} \xi(s) \mathrm{d} s-c \int_{\hat{h}}^{h} e^{|\beta|(2 \hat{h}-y-s)} \xi(s) \mathrm{d} s+2|\beta| e^{|\beta|(\hat{h}-y)} u(\hat{h})\right] .
$$

Lemma 5.9. Let $\boldsymbol{p}=\left(p_{1}, p_{2}, p_{3}\right)^{\top}$ be the solution of (5.23). Then for sufficiently large $|n|_{\max }$, the following estimate holds:

$$
\left|p_{j n}(h)\right| \lesssim|n|_{\max } e^{\left|\beta_{2 n}\right|(\hat{h}-h)} \sum_{j=1,2,3}\left|p_{j n}(\hat{h})\right|+\frac{1}{|n|_{\max }} \sum_{j=1,2,3}\left\|\xi_{j n}\right\|_{L^{\infty}([\hat{h}, h])},
$$

where $p_{j n}$ are the Fourier coefficients of $p_{j}, j=1,2,3$. 
Proof. Let $c_{1}=1 /(\lambda+2 \mu)$ and $c_{2}=1 / \mu$. We solve the two-point boundary value problems of (5.27), (5.28) and get the solutions

$$
\begin{aligned}
g_{n}\left(x_{3}\right)= & \frac{1}{2\left|\beta_{1 n}\right|}\left[-c_{1} \int_{h}^{x_{3}} e^{\left|\beta_{1 n}\right|\left(x_{3}-s\right)} \zeta_{n}(s) \mathrm{d} s+c_{1} \int_{\hat{h}}^{x_{3}} e^{\left|\beta_{1 n}\right|\left(s-x_{3}\right)} \zeta_{n}(s) \mathrm{d} s\right. \\
& \left.-c_{1} \int_{\hat{h}}^{h} e^{\left|\beta_{1 n}\right|\left(2 \hat{h}-x_{3}-s\right)} \zeta_{n}(s) \mathrm{d} s+2\left|\beta_{1 n}\right| e^{\left|\beta_{1 n}\right|\left(\hat{h}-x_{3}\right)} g_{n}(\hat{h})\right], \\
q_{1 n}\left(x_{3}\right)= & \frac{1}{2\left|\beta_{2 n}\right|}\left[-c_{2} \int_{h}^{x_{3}} e^{\left|\beta_{2 n}\right|\left(x_{3}-s\right)} Z_{1 n}(s) \mathrm{d} s+c_{2} \int_{\hat{h}}^{x_{3}} e^{\left|\beta_{2 n}\right|\left(s-x_{3}\right)} Z_{1 n}(s) \mathrm{d} s\right. \\
& \left.-c_{2} \int_{\hat{h}}^{h} e^{\left|\beta_{2 n}\right|\left(2 \hat{h}-x_{3}-s\right)} Z_{1 n}(s) \mathrm{d} s+2\left|\beta_{2 n}\right| e^{\left|\beta_{2 n}\right|\left(\hat{h}-x_{3}\right)} q_{1 n}(\hat{h})\right], \\
q_{2 n}\left(x_{3}\right)= & \frac{1}{2\left|\beta_{2 n}\right|}\left[-c_{2} \int_{h}^{x_{3}} e^{\left|\beta_{2 n}\right|\left(x_{3}-s\right)} Z_{2 n}(s) \mathrm{d} s+c_{2} \int_{\hat{h}}^{x_{3}} e^{\left|\beta_{2 n}\right|\left(s-x_{3}\right)} Z_{2 n}(s) \mathrm{d} s\right. \\
& \left.-c_{2} \int_{\hat{h}}^{h} e^{\left|\beta_{2 n}\right|\left(2 \hat{h}-x_{3}-s\right)} Z_{2 n}(s) \mathrm{d} s+2\left|\beta_{2 n}\right| e^{\left|\beta_{2 n}\right|\left(\hat{h}-x_{3}\right)} q_{2 n}(\hat{h})\right] .
\end{aligned}
$$

Taking the derivatives of (5.33)-(5.35) and then evaluating at $x_{3}=\hat{h}$ gives

$$
\begin{aligned}
q_{1 n}^{\prime}(\hat{h}) & =c_{2} \int_{\hat{h}}^{h} e^{\left|\beta_{2 n}\right|(\hat{h}-s)} Z_{1 n}(s) \mathrm{d} s-\left|\beta_{2 n}\right| q_{1 n}(\hat{h}), \\
q_{2 n}^{\prime}(\hat{h}) & =c_{2} \int_{\hat{h}}^{h} e^{\left|\beta_{2 n}\right|(\hat{h}-s)} Z_{2 n}(s) \mathrm{d} s-\left|\beta_{2 n}\right| q_{2 n}(\hat{h}), \\
g_{n}^{\prime}(\hat{h}) & =c_{1} \int_{\hat{h}}^{h} e^{\left|\beta_{1 n}\right|(\hat{h}-s)} \zeta_{n}(s) \mathrm{d} s-\left|\beta_{1 n}\right| g_{n}(\hat{h}) .
\end{aligned}
$$

Evaluating (5.31) at $x_{3}=\hat{h}$ and then using (5.36)-(5.37), we get

$$
\begin{aligned}
q_{3 n}(\hat{h})= & -\frac{\mathrm{i} \alpha_{1 n}}{\left|\beta_{2 n}\right|} q_{1 n}(\hat{h})-\frac{\mathrm{i} \alpha_{2 n}}{\left|\beta_{2 n}\right|} q_{2 n}\left(\hat{h}_{2}\right)+\frac{1}{\left|\beta_{2 n}\right|^{2}} \frac{1}{\mu} Z_{3 n}(\hat{h}) \\
& +\frac{\mathrm{i} \alpha_{1 n}}{\left|\beta_{2 n}\right|^{2}} c_{2} \int_{\hat{h}}^{h} e^{\left|\beta_{2 n}\right|(\hat{h}-s)} Z_{1 n}(s) \mathrm{d} s+\frac{\mathrm{i} \alpha_{2 n}}{\left|\beta_{2 n}\right|^{2}} c_{2} \int_{\hat{h}}^{h} e^{\left|\beta_{2 n}\right|(\hat{h}-s)} Z_{2 n}(s) \mathrm{d} s
\end{aligned}
$$

Plugging (5.25), (5.26) and (5.31) into (5.29) yields

$$
\left(p_{1 n}(h), p_{2 n}(h), p_{3 n}(h)\right)^{\top}=\frac{1}{\left|\beta_{2 n}\right|} K_{n}\left(q_{1 n}(\hat{h}), q_{2 n}(\hat{h}), g_{n}(\hat{h})\right)^{\top},
$$

where

$$
K_{n}=\left[\begin{array}{ccc}
\alpha_{1 n} \alpha_{2 n} & \left|\beta_{2 n}\right|^{2}+\alpha_{2 n}^{2} & \mathrm{i} \alpha_{1 n}\left|\beta_{2 n}\right| \\
-\left|\beta_{2 n}\right|^{2}-\alpha_{1 n}^{2} & -\alpha_{1 n} \alpha_{2 n} & \mathrm{i} \alpha_{2 n}\left|\beta_{2 n}\right| \\
-\mathrm{i} \alpha_{2 n}\left|\beta_{2 n}\right| & \mathrm{i} \alpha_{1 n}\left|\beta_{2 n}\right| & -\left|\beta_{1 n}\right|\left|\beta_{2 n}\right|
\end{array}\right] .
$$

It follows from a straightforward calculation that the inverse of $K_{n}$ is

$$
K_{n}^{-1}=\frac{1}{\left|\beta_{2 n}\right|^{2} \chi_{n}\left(\left|\beta_{2 n}\right|^{2}+\left|\boldsymbol{\alpha}_{n}\right|^{2}\right)} \hat{K}_{n}
$$

where the entries of the matrix $\hat{K}_{n}$ are

$$
\hat{K}_{11}^{(n)}=\alpha_{1 n} \alpha_{2 n}\left|\beta_{2 n}\right|\left(\left|\beta_{1 n}\right|+\left|\beta_{2 n}\right|\right), \quad \hat{K}_{13}^{(n)}=\mathrm{i} \alpha_{2 n}\left|\beta_{2 n}\right|\left(\left|\beta_{2 n}\right|^{2}+\left|\boldsymbol{\alpha}_{n}\right|^{2}\right),
$$




$$
\begin{aligned}
& \hat{K}_{12}^{(n)}=-\alpha_{1 n}^{2}\left|\beta_{2 n}\right|^{2}+\left|\beta_{1 n}\right|\left|\beta_{2 n}\right|^{3}+\alpha_{2 n}^{2}\left|\beta_{1 n}\right|\left|\beta_{2 n}\right|, \\
& \hat{K}_{21}^{(n)}=\alpha_{2 n}^{2}\left|\beta_{2 n}\right|^{2}-\left|\beta_{1 n}\right|\left|\beta_{2 n}\right|^{3}-\alpha_{1 n}^{2}\left|\beta_{1 n}\right|\left|\beta_{2 n}\right|, \\
& \hat{K}_{22}^{(n)}=-\alpha_{1 n} \alpha_{2 n}\left|\beta_{2 n}\right|\left(\left|\beta_{1 n}\right|+\left|\beta_{2 n}\right|\right), \quad \hat{K}_{23}^{(n)}=-\mathrm{i} \alpha_{1 n}\left|\beta_{2 n}\right|\left(\left|\beta_{2 n}\right|^{2}+\left|\boldsymbol{\alpha}_{n}\right|^{2}\right), \\
& \hat{K}_{31}^{(n)}=-\mathrm{i} \alpha_{1 n}\left|\beta_{2 n}\right|\left(\left|\beta_{2 n}\right|^{2}+\left|\boldsymbol{\alpha}_{n}\right|^{2}\right), \quad \hat{K}_{32}^{(n)}=-\mathrm{i} \alpha_{2 n}\left|\beta_{2 n}\right|\left(\left|\beta_{2 n}\right|^{2}+\left|\boldsymbol{\alpha}_{n}\right|^{2}\right), \\
& \hat{K}_{33}^{(n)}=\left|\beta_{2 n}\right|^{2}\left(\left|\beta_{2 n}\right|^{2}+\left|\boldsymbol{\alpha}_{n}\right|^{2}\right) .
\end{aligned}
$$

Evaluating (5.33)-(5.35) at $x_{3}=h$, we get

$$
\left[\begin{array}{c}
q_{1 n}(h) \\
q_{2 n}(h) \\
g_{n}(h)
\end{array}\right]=\left[\begin{array}{ccc}
e^{\left|\beta_{2 n}\right|(\hat{h}-h)} & 0 & 0 \\
0 & e^{\left|\beta_{2 n}\right|(\hat{h}-h)} & 0 \\
0 & 0 & e^{\left|\beta_{1 n}\right|(\hat{h}-h)}
\end{array}\right]\left[\begin{array}{c}
q_{1 n}(\hat{h}) \\
q_{2 n}(\hat{h}) \\
g_{n}(\hat{h})
\end{array}\right]+\left[\begin{array}{c}
\hat{w}_{1 n} \\
\hat{w}_{2 n} \\
\hat{w}_{3 n}
\end{array}\right],
$$

where

$$
\begin{aligned}
& \hat{w}_{1 n}=\frac{c_{2}}{2\left|\beta_{2 n}\right|}\left[\int_{\hat{h}}^{h} e^{\left|\beta_{2 n}\right|(s-h)} Z_{1 n}(s) \mathrm{d} s-\int_{\hat{h}}^{h} e^{\left|\beta_{2 n}\right|(2 \hat{h}-h-s)} Z_{1 n}(s) \mathrm{d} s\right], \\
& \hat{w}_{2 n}=\frac{c_{2}}{2\left|\beta_{2 n}\right|}\left[\int_{\hat{h}}^{h} e^{\left|\beta_{2 n}\right|(s-h)} Z_{2 n}(s) \mathrm{d} s-\int_{\hat{h}}^{h} e^{\left|\beta_{2 n}\right|(2 \hat{h}-h-s)} Z_{2 n}(s) \mathrm{d} s\right], \\
& \hat{w}_{3 n}=\frac{c_{1}}{2\left|\beta_{1 n}\right|}\left[\int_{\hat{h}}^{h} e^{\left|\beta_{1 n}\right|(s-h)} \zeta_{n}(s) \mathrm{d} s-\int_{\hat{h}}^{h} e^{\left|\beta_{1 n}\right|(2 \hat{h}-h-s)} \zeta_{n}(s) \mathrm{d} s\right] .
\end{aligned}
$$

Similarly, we evaluate (5.29) at $x_{3}=\hat{h}$ and get

$$
\left[\begin{array}{c}
p_{1 n}(\hat{h}) \\
p_{2 n}(\hat{h}) \\
p_{3 n}(\hat{h})
\end{array}\right]=\frac{1}{\left|\beta_{2 n}\right|} K_{n}\left[\begin{array}{l}
q_{1 n}(\hat{h}) \\
q_{2 n}(\hat{h}) \\
g_{n}(\hat{h})
\end{array}\right]+\left[\begin{array}{l}
w_{1 n} \\
w_{2 n} \\
w_{3 n}
\end{array}\right]
$$

where

$$
\begin{aligned}
w_{1 n} & =-\frac{1}{\left|\beta_{2 n}\right|^{2}}\left(c_{2} \alpha_{1 n} \alpha_{2 n} \int_{\hat{h}}^{h} e^{\left|\beta_{2 n}\right|(\hat{h}-s)} Z_{1 n}(s) \mathrm{d} s-\mathrm{i} \alpha_{2 n} \frac{1}{\mu} Z_{3 n}(\hat{h})+c_{2}\left(\left|\beta_{2 n}\right|^{2}+\alpha_{2 n}^{2}\right) \int_{\hat{h}}^{h} e^{\left|\beta_{2 n}\right|(\hat{h}-s)} Z_{2 n}(s) \mathrm{d} s\right), \\
w_{2 n} & =\frac{1}{\left|\beta_{2 n}\right|^{2}}\left(c_{2} \alpha_{1 n} \alpha_{2 n} \int_{\hat{h}}^{h} e^{\left|\beta_{2 n}\right|(\hat{h}-s)} Z_{2 n}(s) \mathrm{d} s-\mathrm{i} \alpha_{1 n} \frac{1}{\mu} Z_{3 n}(\hat{h})+c_{2}\left(\left|\beta_{2 n}\right|^{2}+\alpha_{1 n}^{2}\right) \int_{\hat{h}}^{h} e^{\left|\beta_{2 n}\right|(\hat{h}-s)} Z_{1 n}(s) \mathrm{d} s\right), \\
w_{3 n} & =c_{1} \int_{\hat{h}}^{h} e^{\left|\beta_{1 n}\right|(\hat{h}-s)} \zeta_{n}(s) \mathrm{d} s .
\end{aligned}
$$

It follows from (5.41) that we have

$$
\left[\begin{array}{c}
q_{1 n}(\hat{h}) \\
q_{2 n}(\hat{h}) \\
g_{n}(\hat{h})
\end{array}\right]=\left|\beta_{2 n}\right| K_{n}^{-1}\left[\begin{array}{c}
p_{1 n}(\hat{h}) \\
p_{2 n}(\hat{h}) \\
p_{3 n}(\hat{h})
\end{array}\right]-\left|\beta_{2 n}\right| K_{n}^{-1}\left[\begin{array}{l}
w_{1 n} \\
w_{2 n} \\
w_{3 n}
\end{array}\right] .
$$


Substituting (5.42) into (5.40) leads to

$$
\begin{aligned}
{\left[\begin{array}{l}
p_{1 n}(h) \\
p_{2 n}(h) \\
p_{3 n}(h)
\end{array}\right] } & =\frac{1}{\left|\beta_{2 n}\right|} K_{n}\left[\begin{array}{c}
q_{1 n}(h) \\
q_{2 n}(h) \\
g_{n}(h)
\end{array}\right] \\
& =\frac{1}{\left|\beta_{2 n}\right|} K_{n}\left[\begin{array}{ccc}
e^{\left|\beta_{2 n}\right|(\hat{h}-h)} & 0 & 0 \\
0 & e^{\left|\beta_{2 n}\right|(\hat{h}-h)} & 0 \\
0 & 0 & e^{\left|\beta_{1 n}\right|(\hat{h}-h)}
\end{array}\right]\left[\begin{array}{c}
q_{1 n}(\hat{h}) \\
q_{2 n}(\hat{h}) \\
g_{n}(\hat{h})
\end{array}\right]+\frac{1}{\left|\beta_{2 n}\right|} K_{n}\left[\begin{array}{c}
\hat{w}_{1 n} \\
\hat{w}_{2 n} \\
\hat{w}_{3 n}
\end{array}\right] .
\end{aligned}
$$

Plugging (5.44) into the above equation gives

$$
\left[\begin{array}{l}
p_{1 n}(h) \\
p_{2 n}(h) \\
p_{3 n}(h)
\end{array}\right]=P_{n}\left[\begin{array}{l}
p_{1 n}(\hat{h}) \\
p_{2 n}(\hat{h}) \\
p_{3 n}(\hat{h})
\end{array}\right]-P_{n}\left[\begin{array}{l}
w_{1 n} \\
w_{2 n} \\
w_{3 n}
\end{array}\right]+\frac{1}{\left|\beta_{2 n}\right|} K_{n}\left[\begin{array}{l}
\hat{w}_{1 n} \\
\hat{w}_{2 n} \\
\hat{w}_{3 n}
\end{array}\right]
$$

where the matrix $P_{n}$ is given in (5.7).

Following the same proof as that for Lemma 5.8 of [32], we may show that

$$
\left|\hat{w}_{j n}\right| \lesssim \frac{1}{|n|_{\max }^{2}} \sum_{j=1,2,3}\left\|\xi_{j n}\right\|_{L^{\infty}([\hat{h}, h])}, \quad\left|w_{j n}\right| \lesssim \frac{1}{|n|_{\max }^{2}} \sum_{j=1,2,3}\left\|\xi_{j n}\right\|_{L^{\infty}([\hat{h}, h])} .
$$

By (5.10) and (5.46), we have

$$
\left|\sum_{j=1,2,3} P_{i j}^{(n)} w_{j n}\right| \lesssim \frac{1}{|n|_{\max }} e^{\left(\left|\boldsymbol{\alpha}_{n}\right|-\left|\beta_{2 n}\right|\right)(h-\hat{h})} \sum_{j=1,2,3}\left\|\xi_{j n}\right\|_{L^{\infty}([\hat{h}, h])} .
$$

For sufficiently large $|n|_{\max }$, it is easy to get

$$
\left|\boldsymbol{\alpha}_{n}\right|-\left|\beta_{2 n}\right|=\left|\boldsymbol{\alpha}_{n}\right|-\left(\left|\boldsymbol{\alpha}_{n}\right|^{2}-\kappa_{2}^{2}\right)^{1 / 2}=\frac{\kappa_{2}^{2}}{\left|\boldsymbol{\alpha}_{n}\right|+\left(\left|\boldsymbol{\alpha}_{n}\right|^{2}-\kappa_{2}^{2}\right)^{1 / 2}} \sim \frac{1}{|n|_{\max }} .
$$

Plugging the above estimate into (5.47) gives

$$
\left|\sum_{j=1,2,3} P_{i j}^{(n)} w_{j n}\right| \lesssim \frac{1}{|n|_{\max }} \sum_{j=1,2,3}\left\|\xi_{j n}\right\|_{L^{\infty}([\hat{h}, h])} .
$$

It is also easy to check

$$
\left|\frac{1}{\left|\beta_{2 n}\right|} K_{n}\right| \sim O\left(|n|_{\max }\right)
$$

By (5.46), we have

$$
\frac{1}{\left|\beta_{2 n}\right|}\left|\sum_{j=1,2,3} K_{i j}^{(n)} \hat{w}_{j n}\right| \lesssim \frac{1}{|n|_{\max }} \sum_{j=1,2,3}\left\|\xi_{j n}\right\|_{L^{\infty}([\hat{h}, h])},
$$

which completes the proof after substituting (5.10) and (5.48), (5.49) into (5.45). 
Using Lemma 5.9 and the same arguments as those in [32], we may show that

$$
\left|\int_{\Gamma_{h}}\left(T-T_{N}\right) \boldsymbol{\xi} \cdot \overline{\boldsymbol{p}} \mathrm{d} s\right| \lesssim \frac{1}{N}\|\boldsymbol{\xi}\|_{\boldsymbol{H}^{1}(\Omega)}^{2} .
$$

The details are omitted for brevity.

Now we are ready to show the proof of Theorem 5.1.

Proof. By (5.3), Lemmas 5.4 and 5.6, we obtain

$$
\begin{aligned}
\|\boldsymbol{\xi}\|_{\boldsymbol{H}^{1}(\Omega)}^{2} \leqslant & C_{1}\left[\left(\sum_{K \in \mathcal{M}_{h}} \eta_{K}^{2}\right)^{1 / 2}+\max _{|n|_{\min }>N}\left(|n|_{\max } e^{-\left|\beta_{2 n}\right|(h-\hat{h})}\right)\left\|\boldsymbol{u}^{\mathrm{inc}}\right\|_{\boldsymbol{H}^{1}(\Omega)}\right. \\
& \left.+\left\|\boldsymbol{u}^{\mathrm{inc}}-\boldsymbol{g}^{h}\right\|_{\boldsymbol{H}^{1 / 2}(S)}\right]\|\boldsymbol{\xi}\|_{\boldsymbol{H}^{1}(\Omega)}+\left(C_{2}+C(\delta)\right)\|\boldsymbol{\xi}\|_{\boldsymbol{L}^{2}(\Omega)}^{2}+\delta\|\boldsymbol{\xi}\|_{\boldsymbol{H}^{1}(\Omega)}^{2},
\end{aligned}
$$

where $C_{1}, C_{2}, C(\delta)$ are positive constants. Choosing a small enough $\delta$ such that $\delta / \min \left(\mu, \omega^{2}\right)<1 / 2$ gives

$$
\begin{aligned}
\|\boldsymbol{\xi}\|_{\boldsymbol{H}^{1}(\Omega)}^{2} \leqslant & 2 C_{1}\left[\left(\sum_{K \in \mathcal{M}_{h}} \eta_{K}^{2}\right)^{1 / 2}+\max _{|n|_{\min }>N}\left(|n|_{\max } e^{-\left|\beta_{2 n}\right|(h-\hat{h})}\right)\left\|\boldsymbol{u}^{\mathrm{inc}}\right\|_{\boldsymbol{H}^{1}(\Omega)}\right. \\
& \left.+\left\|\boldsymbol{u}^{\mathrm{inc}}-\boldsymbol{g}^{h}\right\|_{\boldsymbol{H}^{1 / 2}(S)}\right]\|\boldsymbol{\xi}\|_{\boldsymbol{H}^{1}(\Omega)}+2\left(C_{2}+C(\delta)\right)\|\boldsymbol{\xi}\|_{\boldsymbol{L}^{2}(\Omega)}^{2} .
\end{aligned}
$$

Substituting (5.50) into (5.15) and using Lemma 5.4, we have

$$
\begin{aligned}
\|\boldsymbol{\xi}\|_{\boldsymbol{L}^{2}(\Omega)}^{2} \lesssim & {\left[\left(\sum_{K \in \mathcal{M}_{h}} \eta_{K}^{2}\right)^{1 / 2}+\max _{|n|_{\min }>N}\left(|n|_{\max } e^{-\left|\beta_{2 n}\right|(h-\hat{h})}\right)\left\|\boldsymbol{u}^{\mathrm{inc}}\right\|_{\boldsymbol{H}^{1}(\Omega)}\right.} \\
& \left.+\left\|\boldsymbol{u}^{\mathrm{inc}}-\boldsymbol{g}^{h}\right\|_{\boldsymbol{H}^{1 / 2}(S)}\right]\|\boldsymbol{\xi}\|_{\boldsymbol{H}^{1}(\Omega)}+\frac{1}{N}\|\boldsymbol{\xi}\|_{\boldsymbol{H}^{1}(\Omega)}^{2} .
\end{aligned}
$$

The proof is completed after substituting (5.52) into (5.51) and taking $N$ be a sufficiently large number.

\section{NumericAl EXPERIMENTS}

In this section, we introduce the algorithmic implementation of the adaptive finite element DtN method and present two numerical examples to demonstrate the effectiveness of the proposed method.

\subsection{Adaptive algorithm}

It is shown in Theorem 5.1 that the a posteriori error consists of two parts: the finite element discretization error $\epsilon_{h}$ and the DtN operator truncation error $\epsilon_{N}$, where

$$
\begin{aligned}
\epsilon_{h} & =\left(\sum_{K \in \mathcal{M}_{h}} \eta_{K}^{2}\right)^{1 / 2}+\left\|\boldsymbol{u}^{\mathrm{inc}}-\boldsymbol{g}^{h}\right\|_{\boldsymbol{H}^{1 / 2}(S)}, \\
\epsilon_{N} & =\max _{|n|_{\min }>N}\left(|n|_{\max } e^{-\left|\beta_{2 n}\right|(h-\hat{h})}\right)\left\|\boldsymbol{u}^{\mathrm{inc}}\right\|_{\boldsymbol{H}^{1}(\Omega)} .
\end{aligned}
$$

In the implementation, we choose the parameters $h, \hat{h}$ and $N$ based on (6.1) to make sure that the DtN operator truncation error is smaller than the finite element discretization error. In the following numerical experiments, $\hat{h}$ is chosen such that $\hat{h}=\max _{\boldsymbol{r} \in \mathbb{R}^{2}} f(\boldsymbol{r})$ and $N$ is the smallest positive integer that makes $\epsilon_{N} \leqslant 10^{-8}$. The adaptive finite element DtN algorithm is shown in Table 1. 
TABLE 1. The adaptive finite element DtN method.

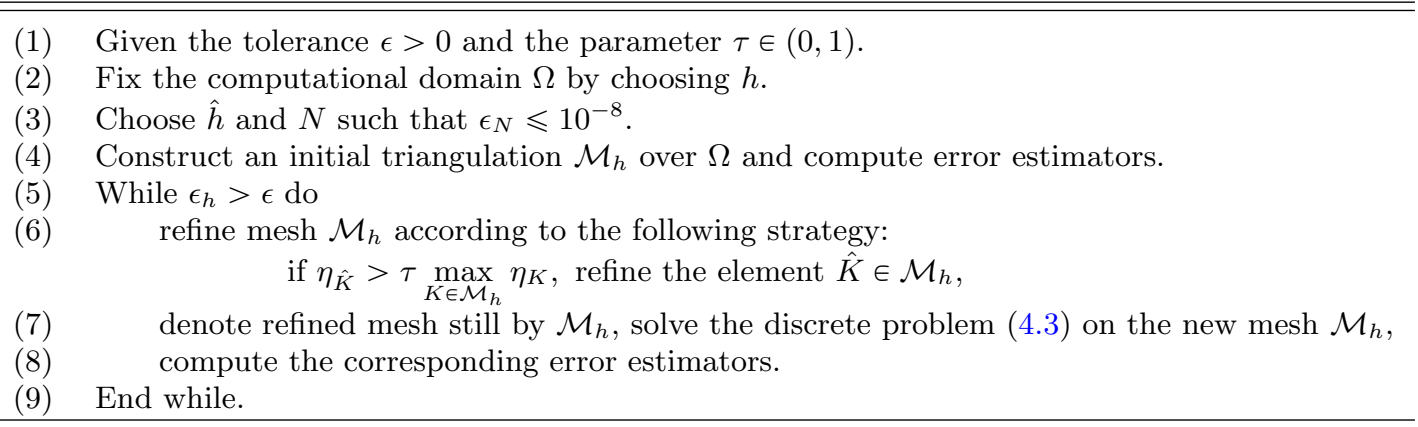

\subsection{Numerical examples}

In this section, we present two examples (cf. [28]) to demonstrate the numerical performance of the DtN method. The first-order linear element is used for solving the problem. Our implementation is based on parallel hierarchical grid (PHG) [36], which is a toolbox for developing parallel adaptive finite element programs on unstructured tetrahedral meshes. The linear system resulted from the finite element discretization is solved by the Supernodal LU (SuperLU) direct solver, which is a general purpose library for the direct solution of large, sparse, nonsymmetric systems of linear equations.

Example 6.1. Consider a simple biperiodic structure, a plane surface, where the exact solution is available. We assume that a plane compressional plane wave $\boldsymbol{u}^{\mathrm{inc}}=\boldsymbol{q} e^{\mathrm{i}\left(\boldsymbol{\alpha} \cdot \boldsymbol{r}-\beta x_{3}\right)}$ is incident on the plane surface $x_{3}=0$, where $\boldsymbol{\alpha}=\left(\alpha_{1}, \alpha_{2}\right)^{\top}, \boldsymbol{q}=\left(q_{1}, q_{2}, q_{3}\right)^{\top}$ with

$$
\begin{aligned}
\alpha_{1} & =\kappa_{1} \sin \theta_{1} \cos \theta_{2}, \alpha_{2}=\kappa_{1} \sin \theta_{1} \sin \theta_{2}, \beta=\kappa_{1} \cos \theta_{1}, \\
q_{1} & =\sin \theta_{1} \cos \theta_{2}, q_{2}=\sin \theta_{1} \sin \theta_{2}, q_{3}=-\cos \theta_{1} .
\end{aligned}
$$

Here $\theta_{1} \in[0, \pi / 2), \theta_{2} \in[0,2 \pi]$ are incident angles. It follows from the elastic wave equation and the Helmholtz decomposition that we may obtain the exact solution for the scattered field

$$
\boldsymbol{u}(\boldsymbol{x})=\mathrm{i}\left[\begin{array}{c}
\alpha_{1} \\
\alpha_{2} \\
\beta
\end{array}\right] a e^{\mathrm{i}\left(\boldsymbol{\alpha} \cdot \boldsymbol{r}+\beta x_{3}\right)}+\mathrm{i}\left[\begin{array}{c}
\alpha_{2} b_{3}-\beta_{20} b_{2} \\
\beta_{20} b_{1}-\alpha_{1} b_{3} \\
\alpha_{1} b_{2}-\alpha_{2} b_{1}
\end{array}\right] e^{\mathrm{i}\left(\boldsymbol{\alpha} \cdot \boldsymbol{r}+\beta_{20} x_{3}\right)},
$$

where

$$
\begin{aligned}
a & =\frac{\mathrm{i}}{\chi}\left(\alpha_{1} q_{1}+\alpha_{2} q_{2}+\beta_{20} q_{3}\right), \\
b_{1} & =\frac{\mathrm{i}}{\chi}\left(\alpha_{1} \alpha_{2}\left(\beta-\beta_{20}\right) q_{1} / \kappa_{2}^{2}+\left(\alpha_{1}^{2} \beta_{20}+\alpha_{2}^{2} \beta+\beta \beta_{20}^{2}\right) q_{2} / \kappa_{2}^{2}-\alpha_{2} q_{3}\right), \\
b_{2} & =\frac{\mathrm{i}}{\chi}\left(-\left(\alpha_{1}^{2} \beta+\alpha_{2}^{2} \beta_{20}+\beta \beta_{20}^{2}\right) q_{1} / \kappa_{2}^{2}-\alpha_{1} \alpha_{2}\left(\beta-\beta_{20}\right) q_{2} / \kappa_{2}^{2}+\alpha_{1} q_{3}\right), \\
b_{3} & =\frac{\mathrm{i}}{\kappa_{2}^{2}}\left(\alpha_{2} q_{1}-\alpha_{1} q_{2}\right),
\end{aligned}
$$

where

$$
\chi=|\boldsymbol{\alpha}|^{2}+\beta \beta_{20}
$$



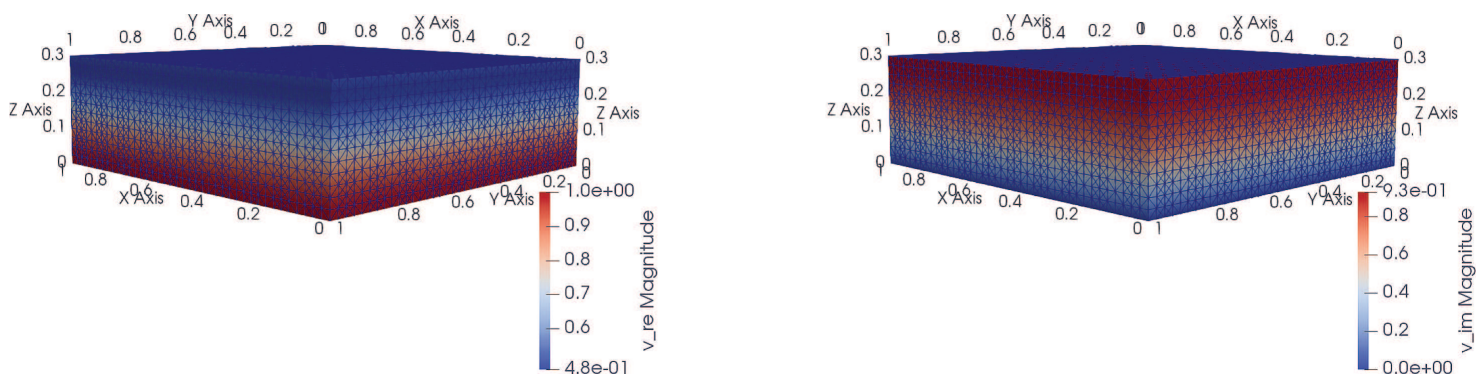

Figure 2. Example 6.1: The mesh and surface plots of the amplitude of the associated solution for the scattered field $\boldsymbol{u}_{h}$. Left: amplitude of the real part of the solution $\left|\Re \boldsymbol{u}_{h}\right|$; right: amplitude of the imaginary part of the solution $\left|\Im u_{h}\right|$.
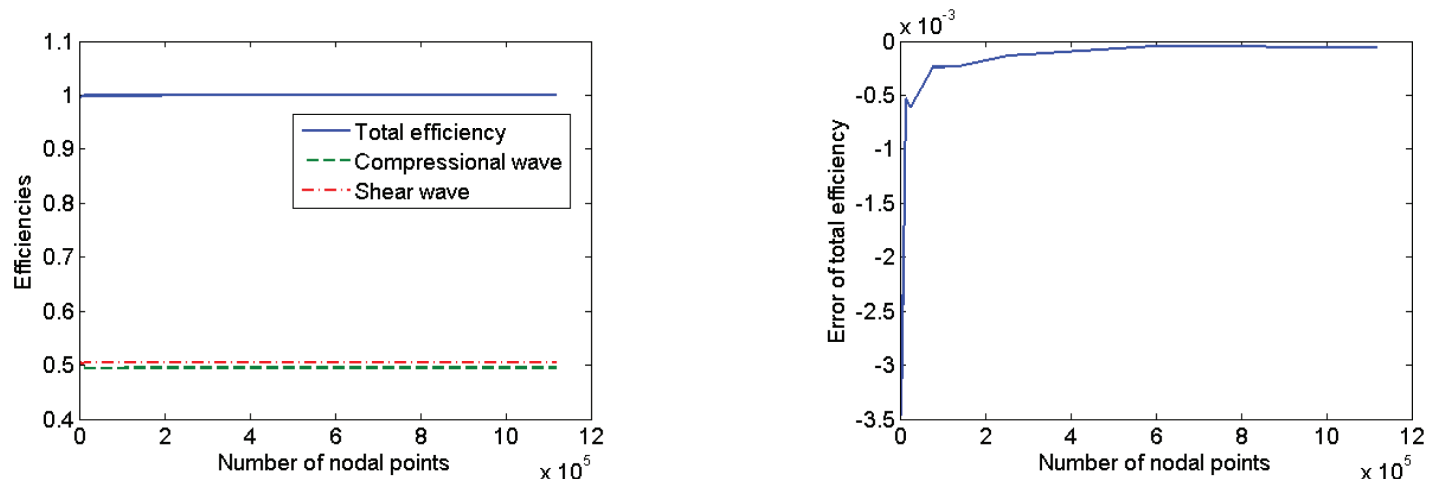

Figure 3. Example 6.1: Left: grating efficiencies; right: error of the grating efficiency.

In our experiments, the parameters are chosen as $\lambda=1, \mu=1, \theta_{1}=\theta_{2}=\pi / 6, \omega=2 \pi$. The computational domain $\Omega=(0,1) \times(0,1) \times(0,0.3)$. The mesh and surface plots of the amplitude of the scattered field $\boldsymbol{v}_{h}$ are shown in Figure 2. The mesh has 228400 tetrahedrons and the total number of degrees of freedom (DoFs) on the mesh is 253200 . The grating efficiencies are displayed in Figure 3, which verifies the conservation of the energy in Theorem 2.1 of [28]. Figure 4 shows the curves of $\log \left\|\nabla\left(\boldsymbol{u}-\boldsymbol{u}_{k}\right)\right\|_{0, \Omega}$ versus $\log N_{k}$ for both the a priori and the a posteriori error estimates with different frequencies, where $N_{k}$ is the total number of DoFs of the mesh. It indicates that the meshes and the associated numerical complexity are quasi-optimal, i.e., $\log \left\|\nabla\left(\boldsymbol{u}-\boldsymbol{u}_{k}\right)\right\|_{0, \Omega}=O\left(N_{k}^{-1 / 3}\right)$ is valid asymptotically. But the results also show that is is less accurate to solve the problem with a higher frequency as the solution becomes more oscillatory.

Example 6.2. This example concerns the scattering of a time-harmonic compressional plane wave $\boldsymbol{u}^{\text {inc }}$ on a flat grating surface with two square bumps, as seen in Figure 5 . The parameters are chosen as $\lambda=1, \mu=1, \theta_{1}=$ $\theta_{2}=\pi / 6, \omega=2 \pi$. The computational domain is $\Omega=(0,1) \times(0,1) \times(0,0.6)$. Since there is no exact solution for this example, we plot in Figure 6 the curves of $\log \left\|\nabla\left(\boldsymbol{u}-\boldsymbol{u}_{k}\right)\right\|_{0, \Omega}$ versus $\log N_{k}$ for the a posteriori error estimates with different frequencies, where $N_{k}$ is the total number of DoFs of the mesh. Again, the result shows that the meshes and the associated numerical complexity are quasi-optimal for the proposed method; but it is less accurate to solve the higher frequency problem. We also plot the grating efficiencies against the DoFs in Figure 7 to verify the conservation of the energy. Figures 8 and 9 show the meshes and the amplitude of the associated solution for the scattered field $\boldsymbol{u}_{h}$ when the mesh has 346734 tetrahedrons. 

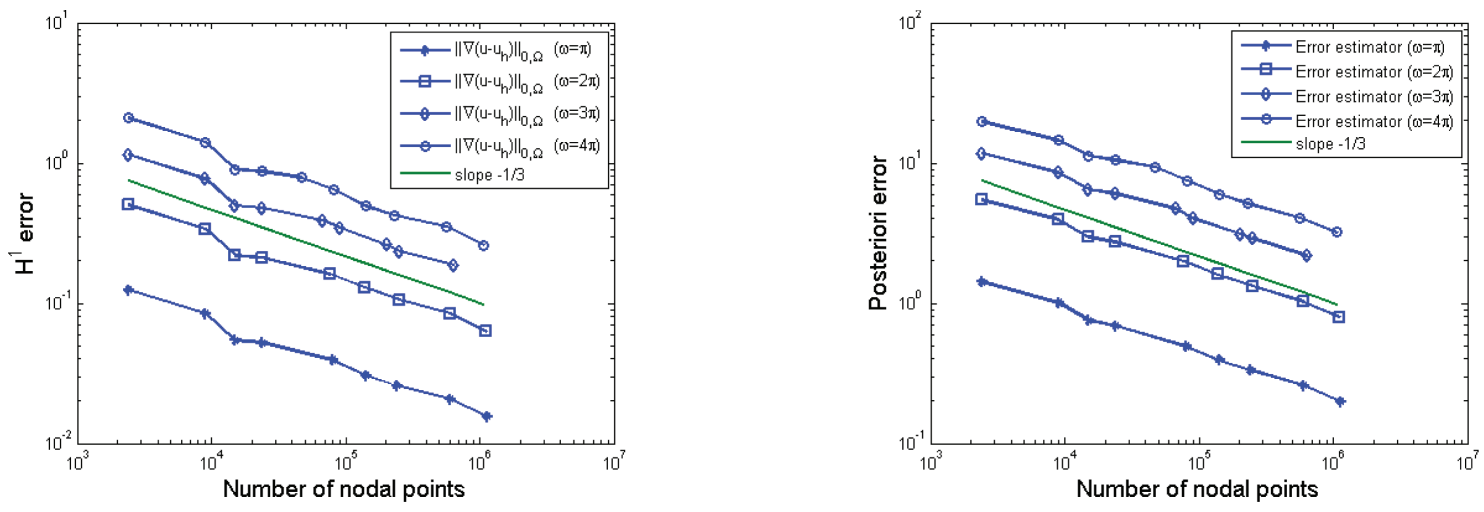

Figure 4. Example 6.1: Left: quasi-optimality of the a priori error estimates; right: quasioptimality of the a posteriori error estimates.

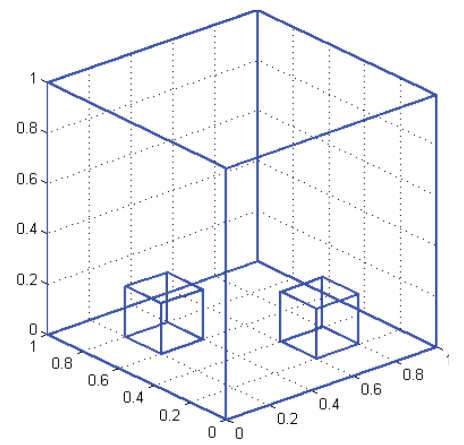

Figure 5. Example 6.2: Problem geometry of the domain.

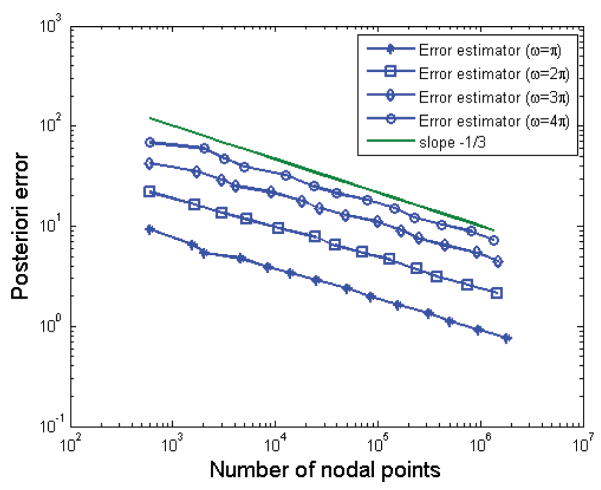

FIgURE 6. Example 6.2: Quasi-optimality of the a posteriori error estimates. 

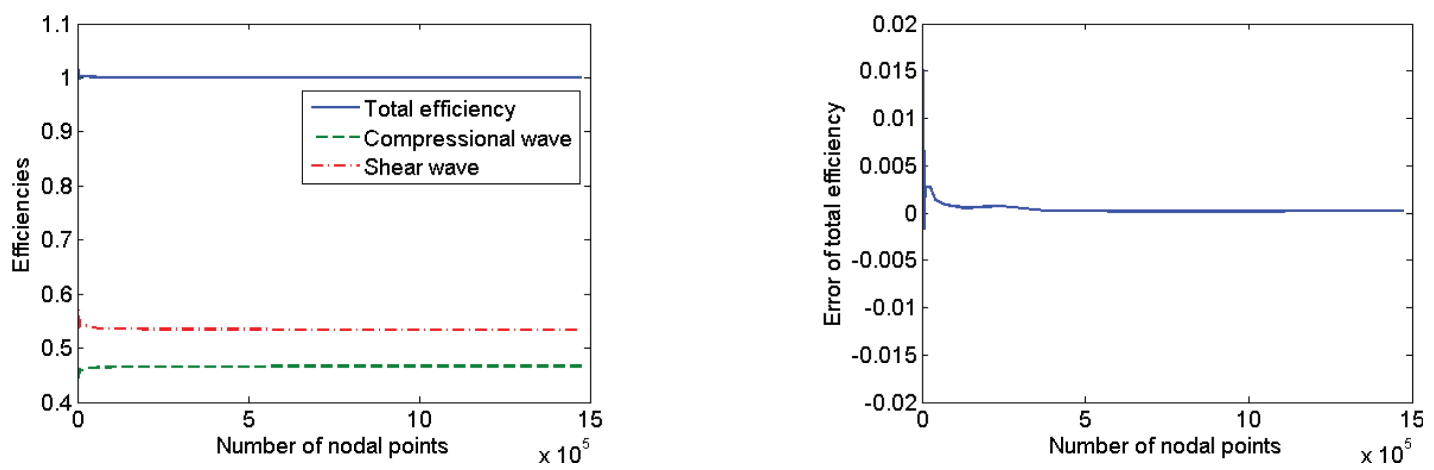

Figure 7. Example 6.2: Left: grating efficiencies; right: error of the grating efficiency.
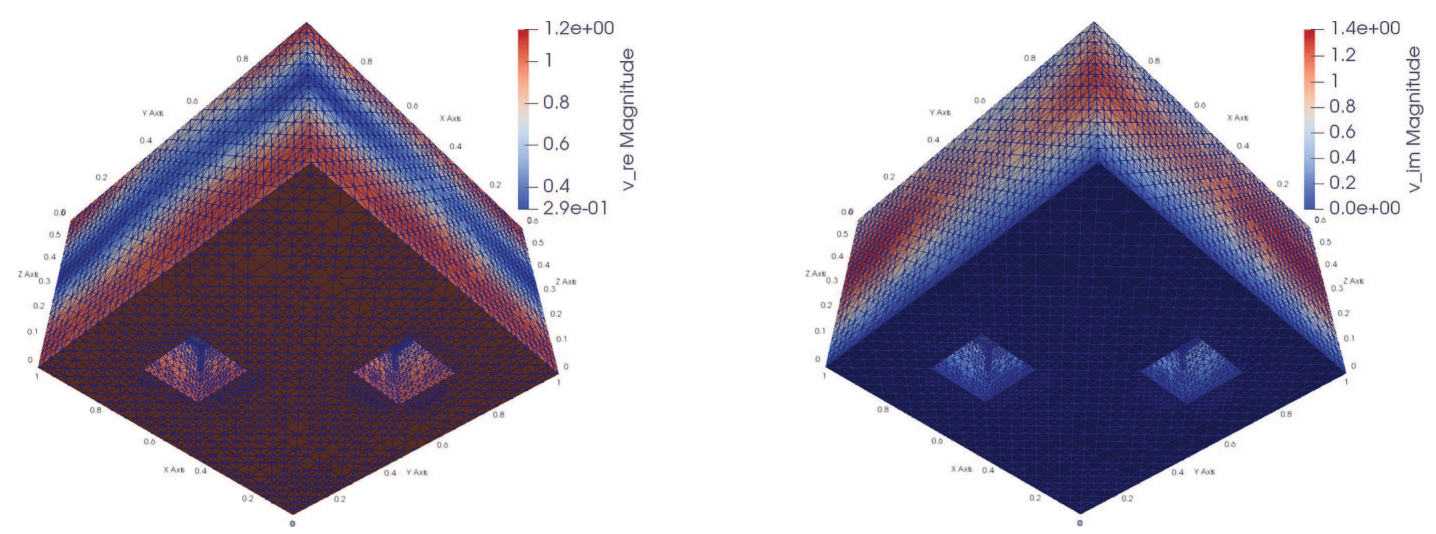

Figure 8. Example 6.2: The mesh and surface plots of the amplitude of the associated solution for the scattered field $\boldsymbol{u}_{h}$ : left: amplitude of the real part of the solution $\left|\Re \boldsymbol{u}_{h}\right|$; right: the amplitude of the imaginary part of the solution $\left|\Im \boldsymbol{u}_{h}\right|$.
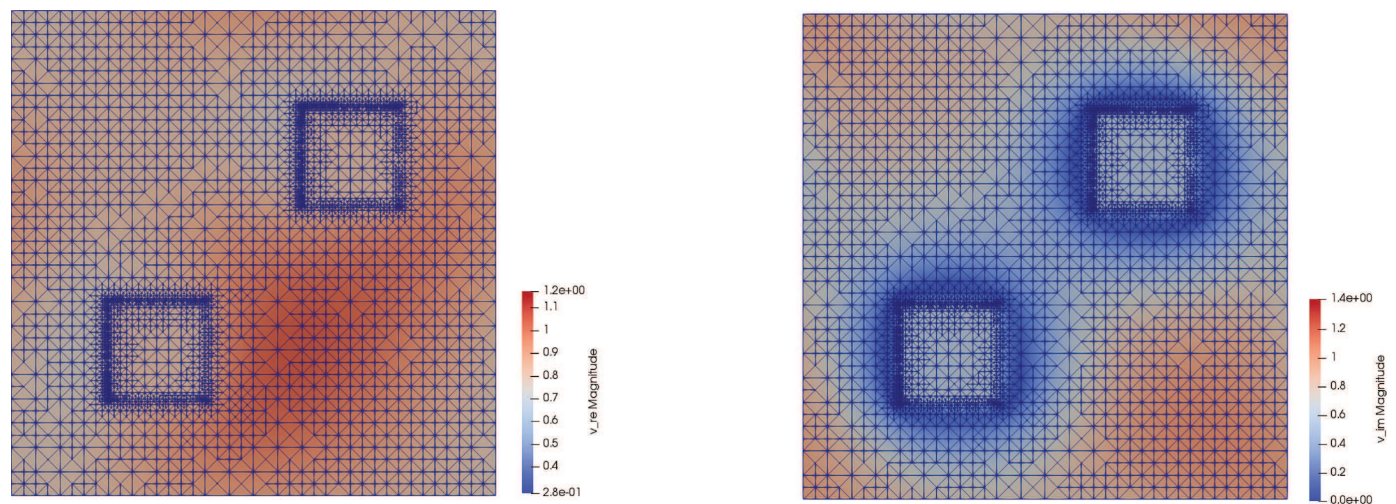

Figure 9. Example 6.2: The mesh and surface plots of the amplitude of the associated solution for the scattered field $\boldsymbol{v}_{h}$ from a view of the $x_{3}$-axis: left: amplitude of the real part of the solution $\left|\Re \boldsymbol{v}_{h}\right|$; right: amplitude of the imaginary part of the solution $\left|\Im \boldsymbol{v}_{h}\right|$. 


\section{Conclusion}

In this paper, we have presented an adaptive finite element DtN method for the elastic scattering problem in bi-periodic structures. Based on the Helmholtz decomposition, a new duality argument is developed to obtain the a posteriori error estimate. It takes account of both the finite element discretization error and the DtN operator truncation error, which is shown to decay exponentially with respect to the truncation parameter. Numerical results show that the proposed method is effective and accurate. This work provides a viable alternative to the adaptive finite element PML method for solving the elastic surface scattering problem. It also enriches the range of choices available for solving elastic wave propagation problems imposed in unbounded domains. Along the line of this work, a possible continuation is to extend our analysis to the adaptive finite element DtN method for solving the three-dimensional obstacle scattering problem and acoustic-elastic interactive problem. The progress will be reported elsewhere on these problems in the future.

Acknowledgements. The work of GB is supported in part by an NSFC Innovative Group Fund (No.11621101). The work of XJ is supported in part by China NSF grants 11771057 and 11671052 . The research of PL is supported partially by the NSF grant DMS-1912704.

\section{REFERENCES}

[1] T. Arens, The scattering of plane elastic waves by a one-dimensional periodic surface. Math. Meth. Appl. Sci. 22 (1999) 55-72.

[2] T. Arens, A new integral equation formulation for the scattering of plane elastic waves by diffraction gratings. J. Integral Equ. Appl. 11 (1999) 275-297.

[3] I. Babuška and A. Aziz, Survey lectures on Mathematical Foundation of the Finite Element Method. In: Mathematical Foundations of the Finite Element Method with Application to the Partial Differential Equations, edited by A. Aziz. Academic Press, New York (1973) 5-359.

[4] I. Babuška and W.C. Rheinboldt, Error estimates for adaptive finite element computations. SIAM J. Numer. Anal. 15 (1978) $736-754$.

[5] G. Bao and H. Wu, On the convergence of the solutions of PML equations for Maxwell's equations. SIAM J. Numer. Anal. 43 (2005) 2121-2143.

[6] G. Bao, P. Li and H. Wu, An adaptive edge element method with perfectly matched absorbing layers for wave scattering by periodic structures. Math. Comput. 79 (2010) 1-34.

[7] J.-P. Bérenger, A perfectly matched layer for the absorption of electromagnetic waves. J. Comput. Phys. 114 (1994) 185-200.

[8] J. H. Bramble and J.E. Pasciak, Analysis of a finite PML approximation for the three dimensional time-harmonic Maxwell and acoustic scattering problems. Math. Comput. 76 (2007) 597-614.

[9] J.H. Bramble, J.E. Pasciak and D. Trenev, Analysis of a finite PML approximation to the three dimensional elastic wave scattering problem. Math. Comput. 79 (2010) 2079-2101.

[10] O. Bruno and F. Reitich, Numerical solution of diffraction problems: a method of variation of boundaries. J. Opt. Soc. Am. A 10 (1993) 1168-1175.

[11] O. Bruno and F. Reitich, Numerical solution of diffraction problems: a method of variation of boundaries II Finitely conducting grating, Padé approximations and singularities. J. Opt. Soc. Am. A 10 (1993) 2307-2316.

[12] J. Chen and Z. Chen, An adaptive perfectly matched layer technique for 3-D time-harmonic electromagnetic scattering problems. Math. Comput. 77 (2008) 673-698.

[13] Z. Chen and X. Liu, An adaptive perfectly matched layer technique for time-harmonic scattering problems. SIAM J. Numer. Anal. 43 (2005) 645-671.

[14] Z. Chen and $\mathrm{H}$. Wu, An adaptive finite element method with perfectly matched absorbing layers for the wave scattering by periodic structures. SIAM J. Numer. Anal. 41 (2003) 799-826.

[15] Z. Chen, X. Xiang and X. Zhang, Convergence of the PML method for elastic wave scattering problems. Math. Comput. 85 (2016) 2687-2714.

[16] W. Chew and W. Weedon, A 3D perfectly matched medium for modified Maxwell's equations with stretched coordinates. Microwave Opt. Tech. Lett. 13 (1994) 599-604.

[17] F. Collino and P. Monk, The perfectly matched layer in curvilinear coordinates. SIAM J. Sci. Comput. 19 (1998) $2061-1090$.

[18] F. Collino and C. Tsogka, Application of the PML absorbing layer model to the linear elastodynamics problem in anisotropic heterogeneous media. Geophysics 66 (2001) 294-307.

[19] W. Dörfler, A convergent adaptive algorithm for Poisson's equation. SIAM J. Numer. Anal. 33 (1996) 1106-1124.

[20] J. Elschner and G. Hu, Scattering of plane elastic waves by three-dimensional diffraction gratings. Math. Meth. Appl. Sci. 22 (2012) 1150019.

[21] D. Givoli and J.B. Keller, Non-reflecting boundary conditions for elastic waves. Wave Motion 12 (1990) 261-279. 
[22] F.D. Hastings, J.B. Schneider and S.L. Broschat, Application of the perfectly matched layer (PML) absorbing boundary condition to elastic wave propagation. J. Acoust. Soc. Am. 100 (1996) 3061-3069.

[23] T. Hohage, F. Schmidt and L. Zschiedrich, Solving time-harmonic scattering problems based on the pole condition. II: convergence of the PML method. SIAM J. Math. Anal. 35 (2003) 547-560.

[24] G.C. Hsiao, N. Nigam, J.E. Pasiak and L. Xu, Error analysis of the DtN-FEM for the scattering problem in acoustic via Fourier analysis. J. Comput. Appl. Math. 235 (2011) 4949-4965.

[25] X. Jiang, P. Li and W. Zheng, Numerical solution of acoustic scattering by an adaptive DtN finite element method. Commun. Comput. Phys. 13 (2013) 1227-1244.

[26] X. Jiang, P. Li, J. Lv and W. Zheng, An adaptive finite element PML method for the elastic wave scattering problem in periodic structures. ESAIM: M2AN 51 (2017) 2017-2047.

[27] X. Jiang, P. Li, J. Lv and W. Zheng, An adaptive finite element method for the wave scattering with transparent boundary condition. J. Sci. Comput. 72 (2017) 936-956.

[28] X. Jiang, P. Li, J. Lv and W. Zheng, Convergence of the PML solution for elastic wave scattering by biperiodic structures. Comm. Math. Sci. 16 (2018) 985-1014.

[29] X. Jiang, P. Li, J. Lv, Z. Wang, H. Wu and W. Zheng, An adaptive finite element DtN method for Maxwell's equation in biperiodic structures. Preprint arXiv: 1811.12449 (2018).

[30] M. Lassas and E. Somersalo, On the existence and convergence of the solution of PML equations. Computing 60 (1998) 229-241.

[31] P. Li and X. Yuan, Convergence of an adaptive finite element DtN method for the elastic wave scattering problem. Preprint arXiv: $1903.03606(2019)$.

[32] P. Li and X. Yuan, Convergence of an adaptive finite element DtN method for the elastic wave scattering by periodic structures. Comput. Methods Appl. Mech. Eng. 360 (2020) 112722.

[33] P. Li, Y. Wang and Y. Zhao, Inverse elastic surface scattering with near-field data. Inverse Prob. 31 (2015) 035009.

[34] P. Monk, A posterior error indicators for Maxwell's equations. J. Comput. Appl. Math. 100 (1998) 173-190.

[35] P. Morin, R. Nochetto and K. Siebert, Convergence of adaptive finite element methods. SIAM Rev. 44 (2002) $631-658$.

[36] PHG (Parallel Hierarchical Grid). http://lsec.cc.ac.cn/phg/

[37] A.H. Schatz, An observation concerning Ritz-Galerkin methods with indefinite bilinear forms. Math. Comput. 28 (1974) 959-962.

[38] R. Verfürth, A Review of A Posterior Error Estimation and Adaptive Mesh Refinement Techniques. Teubner, Stuttgart (1996).

[39] Z. Wang, G. Bao, J. Li, P. Li and H. Wu, An adaptive finite element method for the diffraction grating problem with transparent boundary condition. SIAM J. Numer. Anal. 53 (2015) 1585-1607.

[40] B. Zhang and S.N. Chandler-Wilde, Integral equation methods for scattering by infinite rough surfaces. Math. Methods Appl. Sci. 26 (2003) 463-488.

\section{Subscribe to Open (S20) A fair and sustainable open access model}

This journal is currently published in open access under a Subscribe-to-Open model (S2O). S2O is a transformative model that aims to move subscription journals to open access. Open access is the free, immediate, online availability of research articles combined with the rights to use these articles fully in the digital environment. We are thankful to our subscribers and sponsors for making it possible to publish this journal in open access, free of charge for authors.

\section{Please help to maintain this journal in open access!}

Check that your library subscribes to the journal, or make a personal donation to the S2O programme, by contacting subscribers@edpsciences.org

More information, including a list of sponsors and a financial transparency report, available at: https://www. edpsciences.org/en/maths-s2o-programme 